\title{
Implications of Nonlocal Transport and Conditionally Averaged Statistics on Monin-Obukhov Similarity Theory and Townsend's Attached Eddy Hypothesis
}

\author{
Qi Li AND PIERRE GENTINE \\ Department of Earth and Environmental Engineering, Columbia University, New York, New York \\ JuAn PEDRo MELlado \\ Max Planck Institute for Meteorology, Hamburg, Germany \\ KAIGHIN A. MCCOLL \\ Harvard John A. Paulson School of Engineering and Applied Sciences, and Department of Earth and \\ Planetary Sciences, Harvard University, Cambridge, Massachusetts
}

(Manuscript received 11 October 2017, in final form 5 July 2018)

\begin{abstract}
According to Townsend's hypothesis, so-called wall-attached eddies are the main contributors to turbulent transport in the atmospheric surface layer (ASL). This is also one of the main assumptions of Monin-Obukhov similarity theory (MOST). However, previous evidence seems to indicate that outerscale eddies can impact the ASL, resulting in deviations from the classic MOST scaling. We conduct largeeddy simulations and direct numerical simulations of a dry convective boundary layer to investigate the impact of coherent structures on the ASL. A height-dependent passive tracer enables coherent structure detection and conditional analysis based on updrafts and subsidence. The MOST similarity functions computed from the simulation results indicate a larger deviation of the momentum similarity function $\phi_{m}$ from classical scaling relationships compared to the temperature similarity function $\phi_{h}$. The conditionalaveraged $\phi_{m}$ for updrafts and subsidence are similar, indicating strong interactions between the inner and outer layers. However, $\phi_{h}$ conditioned on subsidence follows the mixed-layer scaling, while its updraft counterpart is well predicted by MOST. Updrafts are the dominant contributors to the transport of momentum and temperature. Subsidence, which comprises eddies that originate from the outer layer, contributes increasingly to the transport of temperature with increasing instability. However, $u^{\prime}$ of different signs are distributed symmetrically in subsidence unlike the predominantly negative $\theta^{\prime}$ as instability increases. Thus, the spatial patterns of $u^{\prime} w^{\prime}$ differ compared to $\theta^{\prime} w^{\prime}$ in regions of subsidence. These results depict the mechanisms for departure from the MOST scaling, which is related to the stronger role of subsidence.
\end{abstract}

\section{Introduction}

Turbulent exchanges of momentum, temperature, and moisture are essential for quantifying various processes involved in the coupled land-atmosphere system. These processes span a wide range of spatial and temporal scales, ranging from seconds/minutes to seasonal/ interannual (Xue and Shukla 1993; Delire et al. 2004; Notaro 2008; Katul et al. 2007). The state of the surface modifies the partitioning of the surface energy balance and water budgets (Betts and Jakob 2002; Betts 2000;

\footnotetext{
Corresponding author: Qi Li, liqi1026@gmail.com
}

Seneviratne et al. 2010; Gentine et al. 2011a,b), thus mediating the land-atmosphere interactions. In addition, the surface energy partitioning and turbulent exchanges of relevant quantities impact the state of the lower atmosphere, including stability, clouds, precipitation (Gentine et al. 2007; Findell et al. 2011; Gentine et al. 2011a,b, 2013), and especially the wind, temperature, and moisture profiles (Businger et al. 1971; Beljaars and Holtslag 1991; Katul et al. 2011). Applying the Monin-Obukhov similarity theory (MOST; Monin and Obukhov 1954) to quantify the turbulent fluxes thus has far-reaching implications for land-atmosphere interactions. 
MOST states that turbulent processes in the atmospheric surface layer (ASL), over a flat uniform surface in the absence of mean subsidence, can be described by only a few key parameters for horizontally homogeneous and stationary turbulence. These parameters are the friction velocity $u_{*}$, the distance from the ground $z$, the surface temperature flux $Q_{0}$, and the buoyancy parameter $\left(g / T_{v}\right)$, where $g$ is the gravitational acceleration and $T_{v}$ is the reference virtual temperature. The value of $T_{v}$ in the buoyancy parameter is often taken as the surface air temperature, denoted by $T_{0}$ (Foken 2006). Carrying out a dimensional analysis using the Buckingham-Pi theorem, based on these parameters and the free dimensions of time, length, and temperature, MOST states that turbulent processes can be characterized by a single dimensionless stability parameter $z / L$, where $L=-u_{*}^{3} /\left[\kappa\left(g / T_{0}\right) Q_{0}\right]$ is the Obukhov length and $\kappa$ is the von Kármán constant; $|L|$, the Obukhov length, is the height where buoyancy production of turbulent kinetic energy begins to dominate over shear production. The nondimensional forms of the mean wind $\bar{u}$ and scalar $\bar{X}$ (e.g., temperature $T$ or specific humidity $q$ ) gradients are then described as

$$
\begin{aligned}
\frac{\kappa z}{u_{*}} \frac{\partial \bar{u}}{\partial z}=\phi_{m}\left(\frac{z}{L}\right) \quad \text { and } \quad \frac{\kappa z}{T_{*}} \frac{\partial \bar{T}}{\partial z} & =\phi_{h}\left(\frac{z}{L}\right) \quad \text { or } \\
\frac{\kappa z}{q_{*}} \frac{\partial \bar{q}}{\partial z} & =\phi_{q}\left(\frac{z}{L}\right)
\end{aligned}
$$

with $X_{*}$ the scale related to the vertical transport of $X$ (e.g., $u_{*} T_{*}=-\overline{w^{\prime} T^{\prime}}$ ). The functions $\phi_{m}, \phi_{h}$, or $\phi_{q}$ are called similarity relationships/universal functions and must be determined empirically from experimental datasets (Beljaars and Holtslag 1991; Foken 2006) such as the famous Kansas 1968 experiment (Businger et al. 1971). Experiments by Högström (1988) also confirmed the applicability of MOST similar to Businger et al. (1971) and Dyer (1974) after accounting for flow distortion. MOST has been fundamental for advances in a wide range of subjects such as hydrology, micrometeorology, and ecosystem gas exchange. MOST is also useful to provide lower boundary conditions in numerical simulations such as large-eddy simulations (LESs; Moeng 1984; Cancelli et al. 2014), cloud-resolving models, weather forecasting, and climate models. Despite its practical importance, validation from classic field experiments, and recent theoretical justifications of the MOST similarity functions (e.g., Katul et al. 2011; Li et al. 2012; Katul et al. 2013, 2014; Banerjee et al. 2016), there are still open questions concerning the universality of the similarity functions and circumstances when MOST could fail.

Physically, MOST scaling implies that eddies of length scale $z$ and velocity scale $u_{*}$ are the main contributors to the turbulent fluxes and that the eddy diffusivities follow a universal function. Thus, local scaling (i.e., based on local mean gradients of quantities and surface momentum and scalar fluxes) can be regarded as a necessary condition of MOST. However, by using atmospheric surface-layer data of convective plumes, Zhuang (1995) concluded that the vertical advection and transport of turbulent kinetic energy (for height that spans the surface layer to the lower part of the mixed layer; i.e., from $\sim 0.025 z / z_{i}$ to $0.15 z / z_{i}$ ) by turbulence and pressure dominates over the local buoyancy production and viscous dissipation. Since momentum and turbulent kinetic energy are not generated locally, the local turbulent quantities cannot be explained by the local mean flow properties only (Zhuang 1995, p. 1720). This is in contrast with shear-driven turbulence, where there is a local balance between shear production and viscous dissipation to a close approximation. McColl et al. (2017) also showed that the large-scale motions contribute to the buoyancy spectra and they need to be taken into account when using the spectral budget model (Katul et al. 2011) in order for $\phi_{h}$ to match with numerical and observational results.

There have been extensive studies on the coupling between the surface layer and outer regions of the ABL and its implications on MOST (Sun et al. 2016; Khanna and Brasseur 1997, 1998; Johansson et al. 2001). The contribution from large-scale coherent eddies that originate from aloft outside the ASL is recognized as one of the mechanisms for deviations from MOST. Earlier work by Panofsky et al. (1977) showed that in the surface layer the horizontal velocity variance scales with $-z_{i} / L$, where $z_{i}$ is the CBL depth. However, the energy spectra of horizontal velocity components do not collapse when they are plotted using Monin-Obukhov scaling (Kaimal and Finnigan 1994, p. 42). More recently, works by McNaughton et al. (2007) and Laubach and McNaughton (2009) scaled the velocity and temperature spectra using both $z$ and $z_{i}$. They found that both scales are necessary for better collapse of the spectra, pointing to the impact of the outer layer on surface-layer properties. Recent experimental and theoretical work has also explicitly analyzed the role of large coherent eddies, such as observations by Gao et al. (2016) or theory by McColl et al. (2016) on the modulation of ASL fluxes by large eddies.

Numerical studies to study the validity of MOST using LES - with a nested grid configuration to achieve fine resolution near the surface-was first conducted by Khanna and Brasseur (1997) to study the validity of MOST. They found that although $\phi_{m} \sim O(1)$ using MOST variables for normalization, there is systematic variation in $\phi_{m}$ when it is plotted against $z / z_{i}$, suggesting 
an "indirect" influence of $z_{i}$ (Khanna and Brasseur 1997, p. 255), which indicates that $\phi_{m}=\phi_{m}\left(z / L, z / z_{i}\right)$. More recently, the canonical forms of the similarity functions have been assessed based on high-resolution large-eddy simulations by Maronga and Reuder (2017), which were in general agreement with the expected forms of the stability functions, but some discrepancies were observed. For instance, the derived MOST similarity functions clearly demonstrated a within-simulation scatter of the momentum similarity function $\phi_{m}(z / L)$ around the predicted functional relation of $\phi_{m} \sim(-z / L)^{1 / 4}$ (Dyer 1974), although the overall trend of $\phi_{m}$ follows this classic prediction across different simulations. Nevertheless, they reported that the scatter in the temperature and moisture stability correction functions $\left(\phi_{h}\right.$ for temperature or $\phi_{q}$ for moisture) was much less than that of $\phi_{m}$. The larger scatter in $\phi_{m}$ than $\phi_{h}$ has been reported in previous field observations (Salesky and Chamecki 2012). They concluded that such a scatter cannot be explained by random errors of the measurement data only; additional nondimensional parameters need to be included to explain the observed trends. However, Andreas and Hicks (2002) argued that the relatively larger scatter in $\phi_{m}$ is due to the spurious correlation between $\phi_{m}$ and $\zeta$, such that the measurement errors in $u_{*}$ lead to a larger spread of $\phi_{m}$ compared to $\phi_{h}$ when $\phi_{m}$ or $\phi_{h}$ is plotted against $\zeta$. Although the measurement errors are not directly applicable to numerical results, Maronga and Reuder (2017) plotted the $d u / d z$ and $d \theta / d z$ obtained directly from LES versus those obtained from fitted functions, such that $u_{*}$ and $\theta_{*}$ only appear indirectly in the fitted functions to avoid the spurious correlation problem. They found that $\phi_{m}$ still exhibits twice the relative error compared to $\phi_{h}$.

Although high-resolution surface-layer-resolving LES is useful to study the surface layer, there are uncertainties associated with the subgrid-scale models near the surface. Wall-modeled LES remains the conventional approach in the atmospheric sciences community (Moeng 1984; Khanna and Brasseur 1997; Bou-Zeid et al. 2005), where wall models are most often based on MOST itself. These exhibit a tendency to "overshoot" (i.e., exaggerate eddy diffusivity) near the surface (Bou-Zeid et al. 2005; Brasseur and Wei 2010). Although the overshoot can be avoided with a careful choice of simulation parameters (Brasseur and Wei 2010), the situation would be more complex for stratified flows. Direct numerical simulations (DNSs), despite being limited to much smaller Reynolds numbers than realistic ABLs, have also been implemented to examine the boundary layers under nonneutral stability (Chung and Matheou 2012; Shah and Bou-Zeid 2014b; McColl et al. 2017; Pirozzoli et al. 2017) and for studying stratocumulus
(Mellado et al. 2018). While MOST is found to be generally applicable, some discrepancies remain, especially regarding the momentum flux-gradient relationship compared to the canonical prediction, as shown in Pirozzoli et al. (2017). From the perspective of outerand inner-scale interactions, Mellado et al. (2016) demonstrated that under free convective conditions, the free atmospheric stratification impacts the surface layer. Using a spectral budget model approach for the MOST similarity functions and DNS data of a heated channel flow, McColl et al. (2017) found that an additional length scale due to the large-eddy motions, in addition to the distance to the wall $z$, was needed to recover the expected similarity function shape for temperature.

In addition to the emerging evidence from field measurements and numerical simulations regarding the departure from MOST, new conceptual frameworks have been put forth as an alternative model to MOST to explain the observed interactions between outer and inner layers. For instance, the ABL can be viewed as a "complex dynamical system" that consists of organized patterns of motions (McNaughton 2004). Sun et al. (2016) used CASES-99 observations to propose the hockey-stick transition (HOST) hypothesis that emphasizes the disconnection between the local vertical mean gradients and the large-scale nonlocal mixing due to the coherent eddies. The large eddies can be thought of as eddies that follow outer-layer scaling and are often referred as "inactive," while the inner-layer "active" eddies are scaled by distance to the wall $z$ and shear stress $\tau_{0}$ (Townsend 1961; Katul et al. 1996; Townsend 1976). Thus, if the outer-scale eddies indeed interact with the inner-scale eddies, similar to the reasoning of similarity laws in Yaglom (1979), MOST should be modified to include the outer-layer scaling variables, such as $z_{i}$ (the boundary layer height).

Despite the abovementioned conceptual models and evidence of inapplicability of MOST under certain circumstances, it still remains unclear how the interactions across scales can bring about nonlocal transport in an unstable ASL. In addition, Khanna and Brasseur (1997) discussed the fact that mixed-layer eddies that scale on $z_{i}$ are likely influencing the near-surface layer through "sweeping motions" by the largest eddies extending to the surface (Wyngaard 1982, p. 616). However, it is still a challenge to explain how these sweeping motions impact $\phi_{m}$ more than $\phi_{h}$ given the larger scatter in $\phi_{m}$ reported in the literature.

In addition, coherent structures exhibit some important topological changes as stability evolves. Coherent structures transition from hairpin vortex packets (e.g., Hommema and Adrian 2003; Adrian 2007; Perry and Chong 1982; Head and Bandyopadhyay 1981) under 
near-neutral condition into thermals as the buoyancy increases. Results from eddy-covariance measurements have been used to infer the transition ( $\mathrm{Li}$ and Bou-Zeid 2011); LES studies (Khanna and Brasseur 1998; Patton et al. 2016; Salesky et al. 2017) were conducted to examine the transition more closely. Convective roll structures transition into convective cells as a function of $z_{i} / L$ and are associated with the decreasing efficiency of momentum transport compared to scalar transport (Salesky et al. 2017). However, previous studies ( $\mathrm{Li}$ and Bou-Zeid 2011; Patton et al. 2016; Salesky et al. 2017) exclusively relied on quadrant analysis in elucidating the differences in spatial correlations of fluctuating quantities. Few studies have systematically analyzed convective rolls and cells in the context of updraft and subsidence. In addition, how changes in coherent structures are related to the failure of local scaling has not been addressed before. Therefore, in this study, by conducting both high-resolution LES and DNS, we aim to investigate

1) how organized motions in the surface layer of the CBL change with stability and

2) how different types of organized motions (updrafts and subsidence) scale with MOST variables and whether they are consistent with Townsend's attached eddy hypothesis.

\section{Problem formulation in DNS and LES}

\section{a. Numerical setup of DNS}

We use both LES and DNS to mitigate uncertainties in Reynolds number dependence of the DNS and in the subgrid-scale (SGS) model and wall modeling of the LES. For DNS, we solve the incompressible NavierStokes equations with Boussinesq approximation as follows:

$$
\begin{aligned}
\frac{\partial u_{i}}{\partial x_{i}}= & 0 \\
\frac{\partial u_{i}}{\partial t}+u_{j}\left(\frac{\partial u_{i}}{\partial x_{j}}-\frac{\partial u_{j}}{\partial x_{i}}\right)= & -\frac{1}{\rho_{0}} \frac{\partial p^{*}}{\partial x_{i}} \\
& +f_{c}\left(u_{2}-G_{2}\right) \delta_{i 1}-f_{c}\left(u_{1}-G_{1}\right) \delta_{i 2} \\
& +\delta_{i 3} g \frac{\theta-\theta_{\text {ref }}}{\theta_{\text {ref }}}+\nu \frac{\partial^{2} u_{i}}{\partial x_{j} \partial x_{j}} \\
\frac{\partial \theta}{\partial t}+u_{i} \frac{\partial \theta}{\partial x_{i}}= & \alpha \frac{\partial^{2} \theta}{\partial x_{j} \partial x_{j}}
\end{aligned}
$$

where $u_{i}$ is the velocity vector in direction $x_{i}, t$ is time, $p^{*}$ is the modified pressure (more details will be given below), $\varepsilon_{i j k}$ is the permutation tensor, $f_{c}$ is the Coriolis parameter with the dimensional value of $10^{-4} \mathrm{~s}^{-1}$ (corresponding to latitude of $\left.43.4^{\circ}\right), G_{k}$ is the geostrophic wind vector specified as $\left(U_{g}, 0,0\right), \rho_{0}$ is the constant reference density, $g=9.81 \mathrm{~m} \mathrm{~s}^{-2}$ is the gravitational acceleration, $\delta_{i j}$ is the Kronecker delta, $\nu$ is the kinematic viscosity of air, $\theta$ is the potential temperature, $\theta_{\text {ref }}$ is a constant reference potential temperature, and $\alpha$ is the thermal diffusivity. In the equations above, $p^{*}=p+$ (1/2) $\rho_{0} u_{i} u_{i}$, where $p$ is the pressure deviation from the mean pressure field that is in hydrostatic balance and has a steady pressure gradient in the sense of a geostrophic forcing. Coleman et al. (1994) investigated the convective boundary layer using DNS with a similar setup of the equations. The DNS code was adopted from Shah and Bou-Zeid (2014b) and has been validated against Coleman et al. (1994) for mean horizontal winds, temperature, and variance of vertical velocity as well as heat flux by simulating a strongly heated case (their section 5.1) with initial surface Richardson number being -1 and $\operatorname{Re}=G D / \nu=400, D=\left(2 \nu / f_{c}\right)^{1 / 2}$, where $D$ is the laminar Ekman depth (results not shown here).

No-slip and no-penetration velocity boundary conditions are used at the bottom. The top boundary condition uses a free-slip condition with no flow through the boundary. To prevent the reflection of gravity waves at the top of the domain, the top $25 \%$ of the computational domain contains a sponge layer that dissipates the energy (Nieuwstadt et al. 1993). Neumann boundary conditions are used for temperature, with a constant heat flux and zero heat flux for the bottom and top boundaries, respectively. The initial velocity field is given by $u(z)=\left(U_{g}, 0,0\right)$, where $U_{g}$ is the geostrophic wind. The initial temperature field is given as $\theta(z)=\theta_{\text {ref }}$ for $\left(z<z_{i 0}\right)$ and $\theta_{\text {ref }}=\left(z-z_{i 0}\right) \Gamma$, where the lapse rate $\Gamma=0.044 \mathrm{~K} \mathrm{~m}^{-1}$. The initial $z_{i 0}$ is taken as $0.5 L_{z}$, where $L_{z}$ is the height of the domain. The simulations are forced with a mean pressure gradient expressed in terms of the geostrophic wind. The computational domain is uniformly discretized with a staggered grid configuration. The domain is further decomposed into horizontal slices to facilitate parallelization using the message passing interface (MPI). A pseudospectral method is implemented, where the fast Fourier transform (FFT) is used in horizontal directions and second-order finite differences are used in the vertical direction. Using the $3 / 2$ rule (Orszag 1971), nonlinear terms are dealiased by zero padding. Time integration is carried out using the second-order Adams-Bashforth method. Note that only a quasi-equilibrium state can be obtained and the state of the CBL varies with time; thus, the CBL height $z_{i}$, which is determined as the height of the minimum of $\left\langle w^{\prime} \theta^{\prime}\right\rangle$, changes with time. At the end of the simulations, 
$z_{i}$ achieves $60 \%-65 \%$ of the domain height. In this study, $x, y$, and $z$ refer to the streamwise, transverse, and vertical directions. The angle brackets represent spatial averaging in the $x-y$ plane. The prime symbol refers to the deviation from the $x-y$ planar-averaged value unless defined otherwise. All subsequent analyses also use this notation to denote the horizontal spatial averaging. More details of the domain size, resolutions, and parameters of DNS runs are presented in Table 1. Values of $z_{i} / L$ vary with time, and they correspond to the time when we carry out subsequent analyses.

\section{b. Numerical setup of LES}

The LES code (Albertson 1996; Albertson and Parlange 1999) has been validated for neutral and convective boundary layers before (Kumar et al. 2006; Bou-Zeid et al. 2005). The detailed numerical setup is described in Kumar et al. (2006). The numerical scheme of LES is the same as that in DNS, but the governing equations change slightly (since the LES solves the filtered equations), and the divergence of the SGS stress tensor (or SGS heat flux for temperature) appears in the right-hand side of the equations. A scale-dependent
Lagrangian dynamic model (Bou-Zeid et al. 2005) is used as the subgrid-scale model. We adopt a constant subgrid-scale Prandtl number of 0.4 for all scalars since Shah and Bou-Zeid (2014a) showed that using a scaledependent dynamic approach for the subgrid-scale model of scalars only yielded minor differences. Time integration is carried out using the second-order Adams-Bashforth method. The upper boundary condition is stress free with no flow through the boundary. To prevent the reflection of gravity waves from the upper boundary, a damping layer occupying the upper $25 \%$ of the domain is imposed, following the same method as that in DNS runs. The upper thermal boundary condition is zero temperature gradient, and a constant surface heat flux is applied as the bottom boundary condition. Periodic boundary conditions are employed in the horizontal directions. We here use MOST to obtain the instantaneous surface stress as a function of the instantaneous horizontal components of velocity at $d z / 2$, where the functional forms of $\Psi_{m}$ are the same as in Kumar et al. [2006; see their Eqs. (6) and (7)]. The friction velocity $u_{*}^{\mathrm{LES}}$ required to compute the instantaneous surface stress is

$$
\begin{aligned}
u_{*}^{\mathrm{LES}} & =\frac{\kappa \sqrt{u^{2}+v^{2}}}{\ln \left(\frac{z}{z_{0}}\right)-\psi_{m}\left(\frac{z}{L}\right)} \\
\psi_{m} & =\log \left[\left(\frac{1+x^{2}}{2}\right)\left(\frac{1+x}{2}\right)^{2}\right]-2 \tan ^{-1} x+\frac{\pi}{2}, \quad x=\left(1-16 \frac{z}{L}\right)^{1 / 4} .
\end{aligned}
$$

In Eq. (5), $\kappa=0.4$ and the instantaneous components of velocity $u$ and $v$ are test filtered at $2 \Delta$. The test filtering at $2 \Delta$ was found to avoid the logarithmic layer mismatch or the overshoot near the surface (Bou-Zeid et al. 2005). The roughness length $z_{0}$ is at each time step based on the instantaneous value of $u_{*}^{\mathrm{LES}}$ according to $z_{0}=9 \nu / u_{*}^{\mathrm{LES}}$ (Monin and Yaglom 1971, chapter 3; Li et al. 2016), where $\nu=1.50 \times 10^{-5} \mathrm{~m} \mathrm{~s}^{-2}$ is the kinematic viscosity of air. The domain is $1.5 \mathrm{~km}$ in the vertical direction and $2 \pi \mathrm{km}$ in horizontal directions.

The initial velocity field is given by $u(z)=\left(U_{g}, 0,0\right)$, where $U_{g}$ is the geostrophic wind. The initial temperature field is given as $\theta(z)=\theta_{\text {ref }}$ for $\left(z<z_{i 0}\right)$ and $\theta_{\text {ref }}=$ $\left(z-z_{i 0}\right) \Gamma$, where the lapse rate $\Gamma=0.1 \mathrm{~K} \mathrm{~m}^{-1}$. Note that the lapse rates for LES and DNS differ but they give rise to the same nondimensional numbers (more details in the next section). The initial $z_{i 0}$ is taken as $0.5 L_{z}$, where $L_{z}$ is the height of the domain. The simulations are forced with a mean pressure gradient expressed in terms of the geostrophic wind. Note that only a quasi-equilibrium state can be obtained and the state of the CBL varies with time; thus, the CBL height $z_{i}$, which is determined as the height of the minimum of $\left\langle w^{\prime} \theta^{\prime}\right\rangle$, changes with time. At the end of the simulations, $z_{i}$ achieves $60 \%-65 \%$ of the domain height. More details on the domain size, resolutions, and parameters of LES runs are presented in Table 1 . Values of $z_{i} / L$ vary with time, and they correspond to the time when we carry out subsequent analyses.

\section{c. Dimensional analysis}

For the physical system considered here, the external parameters of the problem can be grouped into twodimensional groups, a buoyancy Reynolds number, and a Froude number, as follows:

$$
\operatorname{Re}_{o}=\frac{B_{0}}{\alpha N^{2}}
$$

and 
TABLE 1. Overview of DNS and LES setup, where $z_{i 0}$ is the initial height of CBL depth; $L$ is the Obukhov length; $w_{*}$ is the Deardorff convective velocity scale; $L_{x}, L_{y}$, and $L_{z}$ are the domain sizes in the $x, y$, and $z$ directions, respectively; resolution $\left(n_{x} \times n_{y} \times n_{z}\right)$ for DNS runs is $1200 \times 800 \times 804$ and for LES runs is $512^{3} ; z_{\kappa}$ is defined as $\left(\alpha^{3} / B_{0}\right)^{1 / 4}$. $\operatorname{Re}_{*}=w_{*} z_{i} / \nu=\left(z_{i} / z_{\kappa}\right)^{4 / 3} ; \operatorname{Re}_{\tau}=u_{*} z_{i} / \nu=u_{*} / w_{*}\left(z_{i} / z_{\kappa}\right)^{4 / 3}$; $\mathrm{Ri}_{f}=-B_{0} /\left(\left\langle u^{\prime} w^{\prime}\right\rangle d\langle u\rangle / d z+\left\langle v^{\prime} w^{\prime}\right\rangle d\langle v\rangle / d z\right)$ evaluated $a t z u_{*} / \nu=1$ for DNS and at the height where resolved stress is greater than $95 \%$ of the subgrid component in the surface layer for LES.

\begin{tabular}{|c|c|c|c|c|c|c|c|c|c|c|}
\hline Case & $L_{o} / z_{\kappa}$ & $\mathrm{Fr}$ & $\mathrm{Re}_{*}$ & $\mathrm{Re}_{\tau}$ & $-\mathrm{Ri}_{f}$ & $z_{i 0} / z_{\kappa}$ & $L_{x} / L_{y}$ & $L_{x} / L_{z}$ & $z_{i} / L$ & $w_{*} / u_{*}$ \\
\hline DNS low $B_{0}$ & 15 & 20 & 3555 & 1243 & 0.02 & 472 & 1.5 & 6 & -7.14 & 2.86 \\
\hline DNS high $B_{0}$ & 15 & 5 & 3555 & 554 & 0.62 & 457 & 1.5 & 6 & -105.1 & 6.41 \\
\hline DNS max $B_{0}$ & 15 & 2 & 3681 & 309 & 5.1 & 472 & 1.5 & 6 & -678.2 & 11.9 \\
\hline LES low $B_{0}$ & 15 & 20 & 3838 & 1305 & 0.01 & 472 & 1 & $2 \pi$ & -14.0 & 2.94 \\
\hline LES high $B_{0}$ & 15 & 5 & 3838 & 602 & 0.48 & 457 & 1 & $2 \pi$ & -155 & 6.37 \\
\hline LES $\max B_{0}$ & 15 & 2 & 3838 & 432 & 2.1 & 472 & 1 & $2 \pi$ & -420 & 8.88 \\
\hline
\end{tabular}

$$
\operatorname{Fr}_{s}=\frac{U_{g}}{\sqrt{B_{0} / N}},
$$

where $B_{0}$ is the surface buoyancy flux, $N$ is the BruntVäisälä frequency in the free atmosphere (here taken as vertically homogeneous $), N^{2}=\left(g / \theta_{\text {ref }}\right) \Gamma$, and $\alpha$ is the thermal diffusivity. Notice that $\operatorname{Re}_{o}=\left(L_{o} / z_{\kappa}\right)^{4 / 3}$, where $z_{\kappa}=\left(\alpha^{3} / B_{0}\right)^{1 / 4}$ is the diffusive length scale (Townsend 1959; Mellado et al. 2016; Fedorovich and Shapiro 2009) and $L_{o}=\left(B_{0} / N^{3}\right)^{1 / 2}$, as defined in Garcia and Mellado (2014), represents the height beyond which the stratification of gradient $N^{2}$ impacts the CBL dynamics (Mellado et al. 2016).

Even though molecular viscosity is not an external parameter in the filtered Navier-Stokes equations solved in LES, the effective Reynolds number associated with the subgrid-scale model being "large enough" ensures the accuracy of the resolved turbulence (Sullivan and Patton 2011). We define an equivalent LES run to the DNS as the simulation that is able to achieve the same scale separation between the energy-containing eddies (the outer scale) and the diffusive-length-scale $z_{\kappa}$ for DNS or eddies near the filter cutoff scale for LES (Sullivan and Patton 2011), which are eddies of innerlength scale. In the mixed-layer of the CBL, $z_{i}$ is the characteristic scale of an energy-containing eddy (e.g., Jonker et al. 1999; Kaimal and Finnigan 1994, p. 45). The inner-length scale in LES is determined by the filter scale, which is related to the grid size $\Delta$ for implicitly filtered LES (Rogallo and Moin 1984; Bose et al. 2010). Thus, the equivalent $z_{\kappa}$ in LES up to some constant $\beta$ is given as $z_{\kappa}=\beta \Delta^{f}$, where $\Delta^{f}$ is filter cutoff scale in LES. Note that the value of $\beta$ cannot be determined a priori. More details about dimensional analysis and matching the separation of scales in DNS and LES are presented in appendix C.

\section{d. Height-dependent tracer}

In this study, we adopt a different method for visualizing and diagnosing the coherent structures by introducing a passive tracer $s$ with a dimensionless concentration indicating its height in the computational domain, following Park et al. (2018, 2017, 2016). The initial profile is given as

$$
s\left(z, t=t_{0}\right)=z / z_{i 0},
$$

where $t_{0}$ is the time when we start the tracer simulation. After the desired simulation has reached a quasi-steady state, the tracer is initialized with the concentration profile in Eq. (8). A constant relaxation term $-\left[s-s\left(t=t_{0}\right)\right] / \tau$ is added to the advection-diffusion equation of $s$, where $\tau=n z_{i} / w_{*}$ is the relaxation time scale for some constant $n$, where $w_{*}=\left(z_{i} B_{0}\right)^{1 / 3}$. The relaxation time is used to maintain a quasi-steady mean profile similar to the initial-state $s\left(z, t=t_{0}\right)$. If this term were not added, or equivalently, if $\tau$ tended to infinity, downward accumulation of the tracer would eventually form a well-mixed layer. As a result, one could not track the coherent structures that originate from different levels. Conversely, decreasing $n$ corresponds to retaining a shorter "memory" of the tracers. Thus, only the most active (Couvreux et al. 2010) coherent updrafts and subsidence, departing from their origins recently, would be extracted. However, too short a time scale would remove the memory of the origin (and thus be artificially too localized and too diffusive); thus, an intermediate value has to be chosen. Being able to trace the origins of the passive tracer is the main motivation why we adopt this method. Good convergence was found for $n=1 / 6$, which is then chosen to correctly resolve nonlocal transport while keeping a stratified and nonmixed layer in $s$. In appendix A, we present further details on the effects of this method in identifying the distributions of $s$. Park et al. (2016) conducted sensitivity tests on the time scale and found that this method is robust in extracting the coherent structures and that the optimal range is large, thus minimizing the impact of $n$ on the results. For the best choice of the time-scale $\tau$, we refer the reader to Park et al. (2016, 2018, 2017). 
Note that the updrafts and subsidence selected based on $s$ are different than using other variables such as the vertical velocity $w$ or temperature. If one were to use the sign of $w$ as the criterion for updrafts and downdrafts, as applied in previous work (e.g., Schumann and Moeng 1991a; Khanna and Brasseur 1998), the large variability in $w$ would interfere with correctly identifying coherent structures. Indeed, vertical velocity is usually not a good indicator of coherent structures for several reasons. First, one of the disadvantages of using $w$ as a criterion for selecting a coherent structure in the CBL is because of its noisy structure, as summarized in Berg and Stull (2004, their Table 1; based on a literature review of other studies). In fact, $w$ is low-pass filtered in Patton et al. (2016) to reveal the coherent structures more distinctly; $w^{\prime}$ also has a large magnitude compared to the mean updraft velocity of the thermals (Lenschow and Stephens 1980; Williams and Hacker 1992). Furthermore, $w$ suffers from nonlocal pressure effects, so vorticity or conserved scalars are usually preferred (Wilczek et al. 2012; Okamoto et al. 2007; Farge et al. 2001; Park et al. 2016). Although there are different physical descriptions of turbulent coherent structures (Provenzale 1999; Haller and Yuan 2000), the emerging flow patterns are most easily observed when passive tracers are mixed along the "material lines" (Haller and Yuan 2000), which give rise to the definition of Lagrangian coherent structures. However, this Lagrangian approach is computationally demanding since calculation of the direct Lyapunov component for threedimensional flow fields is required (Green et al. 2007; Karrasch and Haller 2013; Haller 2015; Rockwood et al. 2018). Therefore, the passive tracer with a constant relaxation term adopted here does not add too much computational overhead while keeping a Lagrangian perspective to the coherent structure extraction (Couvreux et al. 2010; Park et al. 2016). Note that the method of proper orthogonal decomposition (Berkooz et al. 1993) has been ubiquitously applied in studying coherent structures in the atmospheric boundary layer (e.g., Huang et al. 2009; Calaf et al. 2013).

The modified advection-diffusion equations for passive tracer $s$ solved in DNS is given by

$$
\frac{\partial s}{\partial t}+u_{i} \frac{\partial s}{\partial x_{i}}=\alpha \frac{\partial^{2} s}{\partial x_{j} \partial x_{j}}-\frac{s-s\left(z, t=t_{0}\right)}{\tau} .
$$

In LES, the corresponding equation is given by

$$
\frac{\partial \tilde{s}}{\partial t}+\widetilde{u}_{i} \frac{\partial \tilde{s}}{\partial x_{i}}=\operatorname{Sc}_{\mathrm{SGS}}^{-1} \nu_{\mathrm{SGS}} \frac{\partial^{2} \tilde{s}}{\partial x_{j} \partial x_{j}}-\frac{\tilde{s}-\tilde{s}\left(t=t_{0}\right)}{\tau},
$$

where $\mathrm{Sc}_{\mathrm{SGS}}$ is the subgrid-scale Schmidt number and $\mathrm{Sc}_{\mathrm{SGS}}=\mathrm{Pr}_{\mathrm{SGS}}=0.4$. The tracer simulation lasts for a total time of $t=z_{i} / 3 w_{*}$. A longer time period $\left(t=2 z_{i} / 3 w_{*}\right)$ is tested, and we find the distributions of $s$ at any particular level show converged results. It is useful to define some functions of the tracer here, which will be extensively used in later sections:

1) The probability density function (pdf) of passive tracer $s(z)$ at height $z$ is denoted by $f(s ; z)$; similarly, $f\left(s^{\prime} ; z\right)$ is the pdf of $s^{\prime}(x, y, z)=s(x, y, z)-\langle s\rangle$.

2) The normalized conditional average for some variable $q$ at a level $z$, given that it has scalar $s$ (and thus that it originates from heights denoted by values of $s$ to the destination $z$ ),

$$
\langle q \mid s ; z\rangle_{0}=\frac{1}{\langle q\rangle} \int_{\text {all } q} q f_{q \mid s}(q \mid s ; z) d q=\frac{\langle q \mid s ; z\rangle}{\langle q\rangle},
$$

where $f_{q \mid s}(q \mid s)$ is the conditional pdf of $q$ given $s$.

3) The weighted conditional average $\left\langle q \mid s^{\prime} ; z\right\rangle_{N}$ is just the normalized conditional average in terms of a fraction:

$$
\left\langle q \mid s^{\prime} ; z\right\rangle_{N}=\frac{f\left(s^{\prime} ; z\right)\left\langle q \mid s^{\prime} ; z\right\rangle}{\langle q\rangle}=f\left(s^{\prime} ; z\right)\left\langle q \mid s^{\prime} ; z\right\rangle_{0},
$$

which represents the relative contribution to $\langle q\rangle$ because $\int_{\text {all } s^{\prime}}\left\langle q \mid s^{\prime} ; z\right\rangle_{N} d s^{\prime}=1$.

We illustrate the capability of this approach in identifying ramp structures, which have been observed in various laboratory and in situ experiments (e.g., Mestayer et al. 1976; Thomas and Foken 2005; Brunet and Irvine 2000; Antonia et al. 1979; Wilczak 1984). A snapshot of the cross sections in the $x-y$ plane at $z / z_{i}=$ 0.08 for the DNS low- $B_{0}$ run is plotted in Fig. 1 . For $s^{\prime}<$ 0 , Fig. 1a shows the updraft regions of $\theta^{\prime} / \theta_{*}$. No numerical values are assigned to the subsidence regions, which appear as white space in Fig. 1a. A similar procedure produces the subsidence regions in Fig. 1 b. Along the black line indicated in Figs. $1 \mathrm{a}$ and $1 \mathrm{~b}$, the red (blue) color in Figs. 1c-e indicates updrafts (subsidence). The ramplike structures in temperature in Fig. $1 \mathrm{c}$ are characterized by a sharp increase in $\theta^{\prime} / \theta_{*}$ in the upwind side (usually referred to as the cliff). It is then followed by a gradual decrease as shown between the magenta vertical lines. Such a cliff-ramp pattern tilted in the upwind direction is consistent with results reported in previous findings in the literature such as Antonia et al. (1979). Signals between the vertical cyan lines show the quiescent baseline temperature. Fewer such quiescent intervals are present with higher convective instability for moderate buoyancy (not shown here), which is consistent with previous observations (Antonia and Chambers 1978). The streamwise long streaks in Figs. $1 \mathrm{a}$ and $1 \mathrm{~b}$ are 


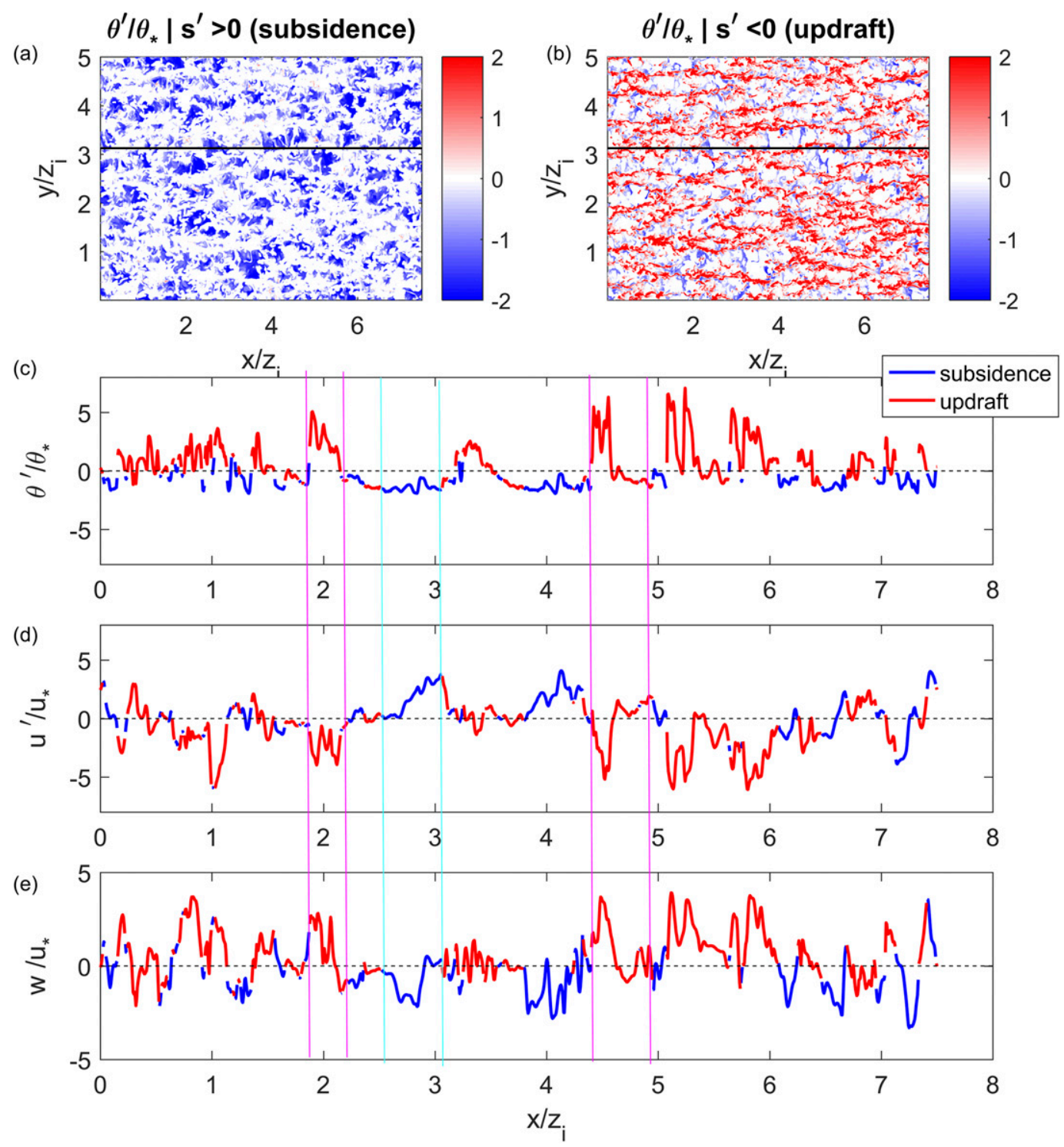

FIG. 1. (a) The $x-y$ cross section of subsidence regions of $\theta^{\prime} / \theta_{*}$. (b) The $x-y$ cross section of updraft regions of $\theta^{\prime} / \theta_{*}$. (c)-(e) Plots of $\theta^{\prime}, u^{\prime}$, and $w$ along the black lines in (a) and (b).

shown to contribute directly to the appearance of ramp structures. The sudden drops (i.e., the cliff in the ramps structures) demarcate the transition between an updraft and a subsidence region. We will apply this approach to examining the conditional statistics and extracting relevant turbulent coherent structures in this study.

\section{Results and discussion}

\section{a. Monin-Obukhov similarity functions}

According to the definition in Stull (1988, p. 10), "the surface layer is the region at the bottom of the boundary layer where turbulent fluxes and stress vary by less than $10 \%$ of their magnitudes." Thus, it is usually defined as the lowest $10 \%$ of the atmospheric boundary layer. In subsequent analyses of the stability functions, $u_{*}$ is defined as the surface stress. In the DNS, it is computed as $u_{*}=\left[\left.(\nu \partial u / \partial z)^{2}\right|_{0}+\left.(\nu \partial v / \partial z)^{2}\right|_{0}\right]^{1 / 4}$, where 0 denotes the gradient taken at the surface. In LES, $u_{*}$ is computed using the wall model based on MOST (Kumar et al. 2006); $\theta_{*}$ is computed from the imposed surface kinematic heat flux and appropriate $u_{*}$. The lower bound of the surface layer is chosen as the height where the turbulent fluxes exceed $95 \%$ of the viscous stress or SGS contribution. The upper bound of the surface layer is 

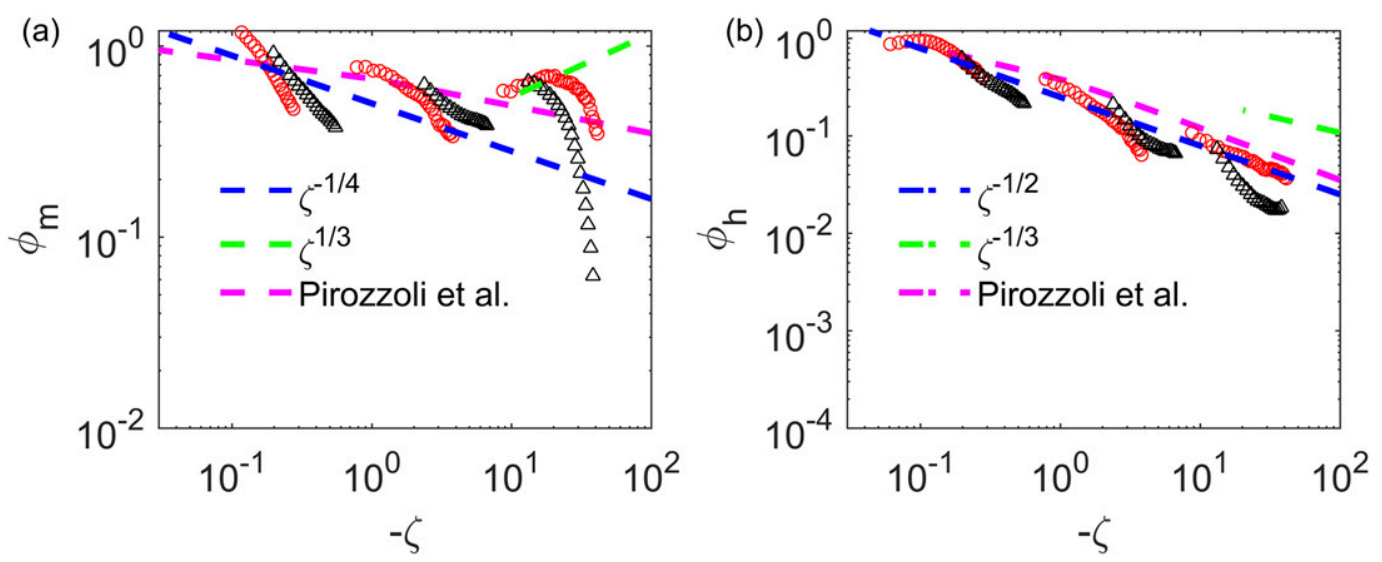

FIG. 2. The stability correction functions for (a) $u$ and (b) $\theta$. Black triangles: DNS data; red circles: LES data. Blue dashed lines refer to the exponent in the classical Businger-Dyer relationships; green dashed lines are the freeconvective limit predicted by Kader and Yaglom (1990); magenta dashed lines are (a) $1 / 0.399(1-14.6 \zeta)^{-0.145}$ and (b) $1 / 0.375(1-5.67 \zeta)^{-0.538}$.

taken as $10 \%$ of $z_{i}$. Note that, in general, this definition is a practical compromise. We proceed with this definition of the surface layer, which is similar to the approach in McColl et al. (2017). We ran the simulations for $1 \mathrm{~h}$ (similar to Maronga and Reuder 2017), which corresponds to $\sim 4-8$ large-eddy turnover times $\left(z_{i} / w_{*}\right)$, before collecting the statistics. The values of $\phi$ in Figs. 2 and 3 are averaged for an additional $1 \mathrm{~h}$. We find that both DNS (black triangles) and LES (red circles) results demonstrate comparable trends, especially for small $\zeta=z / L$, but none of the individual cases follows the predicted power laws according to the classic Businger-Dyer relation of $-1 / 4$ power in $\phi_{m}$ (Fig. 2a) and $-1 / 2$ in $\phi_{h}$ (Fig. 2b). Within a simulation, they all have steeper gradients in the log-log plot than the Businger-Dyer relation. This behavior is in agreement with previous observations in numerical studies (Pirozzoli et al. 2017), which also found discrepancies between the $\phi$ functions and the expected similarity relationship. However, over the range of $\zeta$, which spans more than three decades, the overall trend of $\phi_{h}$ follows the predicted relationship in contrast to the large scatter in $\phi_{m}$. At $-\zeta \geq 8, \phi_{m}$ from the LES run starts to converge to the local free convective limit, where $\phi_{m}=$ $(-\zeta)^{1 / 3}$, which is consistent with the LES results in Maronga and Reuder (2017). For a similar range of $\zeta$, the DNS runs do not exhibit the same transition. The local free-convective limit of $\phi_{h} \sim(-\zeta)^{-1 / 3}$ is not observed for either LES or DNS data, which also supports the reasoning in Businger (1973) about nonzero wind shear due to motions of the buoyancy-driven eddies. Based on this, Businger (1973) predicted a free convective limit scaling of $(-\zeta)^{-1 / 2}$ when $d \theta / d z$ and $d u / d z$ are scaled with $w_{*}=\left(B_{0} z_{i}\right)^{1 / 3}$.
We then investigated similarity functions $\phi$ for the standard deviations of $w$ and $\theta$ in Fig. 3. They are in general in much better agreement with predicted power laws (Mellado et al. 2016; Maronga and Reuder 2017; Pirozzoli et al. 2017) for both the DNS and LES and across stabilities. Interestingly, a more negative exponent in $\zeta$ for $\phi_{\sigma_{\theta}}$ has been demonstrated by previous works and this study, shown in Fig. $3 \mathrm{~b}$. Overall, $\phi_{\sigma_{w}}$ conforms to the MOST scaling better than $\phi_{m}$, which was also reported in numerical simulations by Maronga and Reuder (2017) and Pirozzoli et al. (2017). This may indicate that although $\sigma_{w}$ closely follow MOST-based scaling, the "large-scale circulatory motions" (Pirozzoli et al. 2017, p. 508) caused by the largest eddies extending to the surface with almost pure horizontal motions (Wyngaard 1982) obey a different scaling.

It should be noted that the $\phi$ defined from both DNS and LES are based on numerical models and the results can suffer from limitations of each method. DNS can suffer from the problem of low Re as analyzed in Chung and Matheou (2012) in the similarity functions of a stable boundary layer. They found that the constants in the similarity functions can differ from those obtained from atmospheric measurements. LES is subject to errors in the SGS model as well as surface wall modeling by imposing MOST on the first grid point, which may also lead to inaccuracies in the near-surface profiles. In addition, the resolved flows in LES should be in a regime of "Reynolds number similarity" (Townsend 1976; Wyngaard 2010), and the Reynolds number of the LES solutions depend on the scale separation between the energy-containing eddies and the SGS (Moeng and Wyngaard 1988; Sullivan and Patton 2011). This scale dependence affects the inertial range (Sullivan and Patton 2011) and therefore 

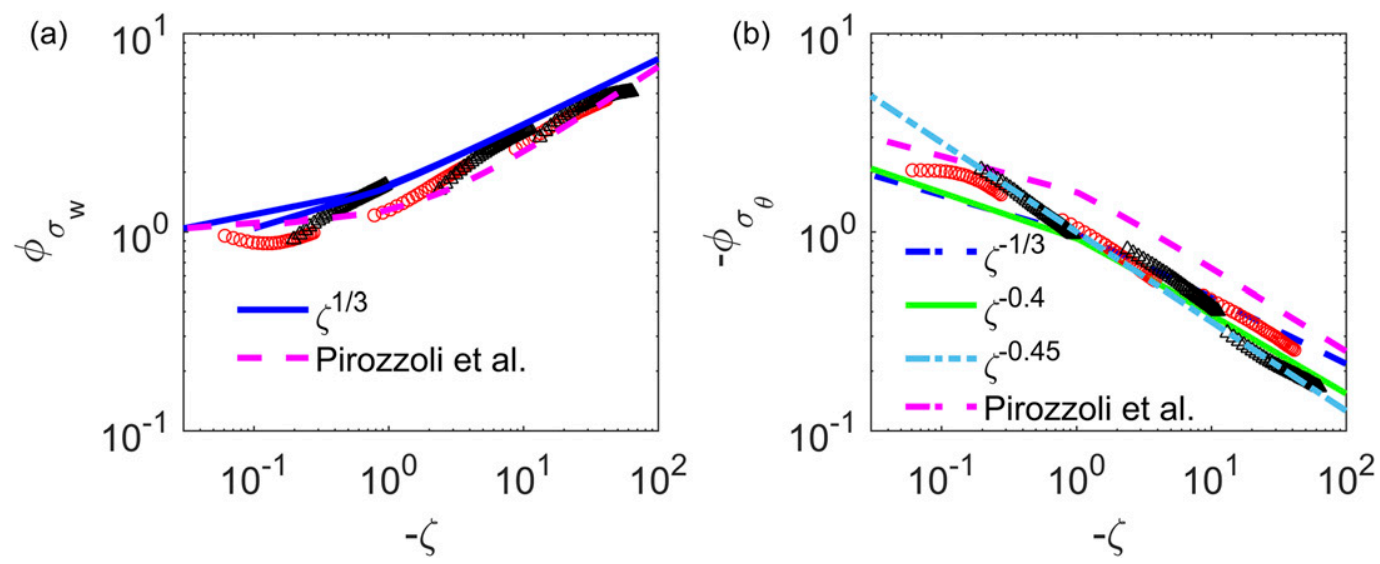

FIG. 3. The stability correction functions for (a) $\sigma_{w}$ and (b) $\sigma_{\theta}$. Black triangles: DNS data; red circles: LES data. Blue solid line in (a): $\phi_{\sigma_{w}}=1.0(1-4.1 \zeta)^{1 / 3}$ (Maronga and Reuder 2017); magenta dotted line in (a): $\phi_{\sigma_{w}}=1 / 1.03(1-$ $0.63 \zeta)^{0.452}$ (Pirozzoli et al. 2017); blue dotted line in (b): $-\phi_{\sigma_{\theta}}=2.1 \times(1-9 \zeta)^{-1 / 3}$ (Maronga and Reuder 2017); green solid line in (b): fitted relation from LES results by Maronga and Reuder (2017): $-\phi_{\sigma_{\theta}}=1.2 \times(0.2-$ $1.7 \zeta)^{-0.4}$; light blue dash-dotted line in (b): fitted relation from DNS results by Mellado et al. (2016): $-\phi_{\sigma_{\theta}}=1.0 \times$ $(-\zeta)^{-0.45}$; magenta dotted line: $-\phi_{\sigma_{\theta}}=1 / 0.318(1-4.08 \zeta)^{-0.420}$ (Pirozzoli et al. 2017).

the surface-layer fluxes and profiles. The consistency of the computed $\phi$ for second-order statistics for both LES and DNS however suggests that DNS can be a viable approach to study the surface layer.

\section{b. Conditionally sampled stability functions}

As mentioned in the introduction, the interactions between the inner and outer layers could contribute to deviations from the classic MOST predictions. To better understand the contribution of outer-layer and inner-layer eddies, we compute the conditionally sampled $\phi_{m}$ and $\phi_{h}$ based on the sign of $s^{\prime}$ to indicate updrafts or subsidence (Fig. 4). Results in Figs. 4 and 5 are averaged when $f(s ; z)$ becomes stationary after the initial transient period, which is approximately $0.066 z_{i} / w_{*}$. We tested doubling the duration of the tracer experiment, and the conclusions about scaling of conditional $\phi_{s}$ do not change. We analyzed $\phi_{m}^{u}\left(\phi_{m}^{d}\right)$, which indicates $\phi_{m}$ is conditioned on updrafts (subsidence). They are computed as $\phi_{m}^{u}=\left(\kappa z / u_{*}\right)\left(d\left\langle u \mid s^{\prime}<0\right\rangle / d z\right)$ $\left[\phi_{m}^{d}=\left(\kappa z / u_{*}\right)\left(d\left\langle u \mid s^{\prime}>0\right\rangle / d z\right)\right] ;$ similar definitions follow for $\phi_{h}^{u}$ and $\phi_{h}^{d}$.

One of the most striking features in Fig. 4 is that $\phi_{h}^{u}$ closely follows the classic scaling of $\sim \zeta^{-1 / 2}$, as indicated by the black line, according to the model in Panofsky and Dutton (1984) and predicted for the free convective limit by Businger (1973), while $\phi_{h}^{d}$ deviates from this predicated scaling (Fig. 4b). Thus, we hypothesize that updrafts and subsidence yield different scaling regimes for temperature fluxes. MOST-based scaling appears reasonable for the updraft contribution to $\phi_{h}$, which can be interpreted as wall-attached eddies. In contrast, $\phi_{h}^{d}$ consists of contributions from large-scale eddies that originate from the outer layer, and $\phi_{h}^{d}$ accounts for most of the observed deviations in Fig. 2b. This finding supports the theoretical insights from McColl et al. (2017) regarding the need to include outer-scale scaling in the cospectral budget model to recover the desired form of $\phi_{h}$.

Unlike $\phi_{h}$, neither $\phi_{m}^{u}$ nor $\phi_{m}^{d}$ follows the expected $\zeta^{-1 / 4}$ scaling, and in fact, both show similar scaling with respect to $\zeta$, especially with increasing convective instability. For example, the slopes in $\phi_{m}^{u}$ and $\phi_{m}^{d}$ become more similar than those in $\phi_{h}^{u}$ and $\phi_{h}^{d}$. Compared to Fig. $4 \mathrm{~d}, \phi_{m}^{u}$ and $\phi_{m}^{d}$ in Fig. $4 \mathrm{c}$ across different stability regimes show a better collapse than $\phi_{h}^{u}$ and $\phi_{h}^{d}$. The dependence on both $z / L$ and $z / z_{i}$ for $u$ supports findings in Khanna and Brasseur (1997). They found using LES that $u$, when scaled according to MOST, showed stronger functional dependence on both $z / L$ and $z / z_{i}$ compared to $\theta$ [a similar conclusion was also reached in Johansson et al. (2001)]. Thus, Figs. 4a, 4c, and 4d together show that $\phi_{m}^{u}$ and $\phi_{m}^{d}$ both depend on $z / L$ and $z / z_{i}$ similarly. This indicates that eddies from the inner and outer regions could interact. In contrast to Townsend's theory of noninteraction between inner- and outer-scale eddies, it appears that even for near-neutral turbulent wall-bounded flow, eddies from the outer layer are shown to penetrate down to the wall (Morrison 2007). One possible mechanism of interaction is via the socalled top-down influence (Hunt and Morrison 2000; Morrison 2007), in which the large-scale eddies above the surface layer impinge at the wall, generating shear stress while decelerating. This mechanism was proposed 

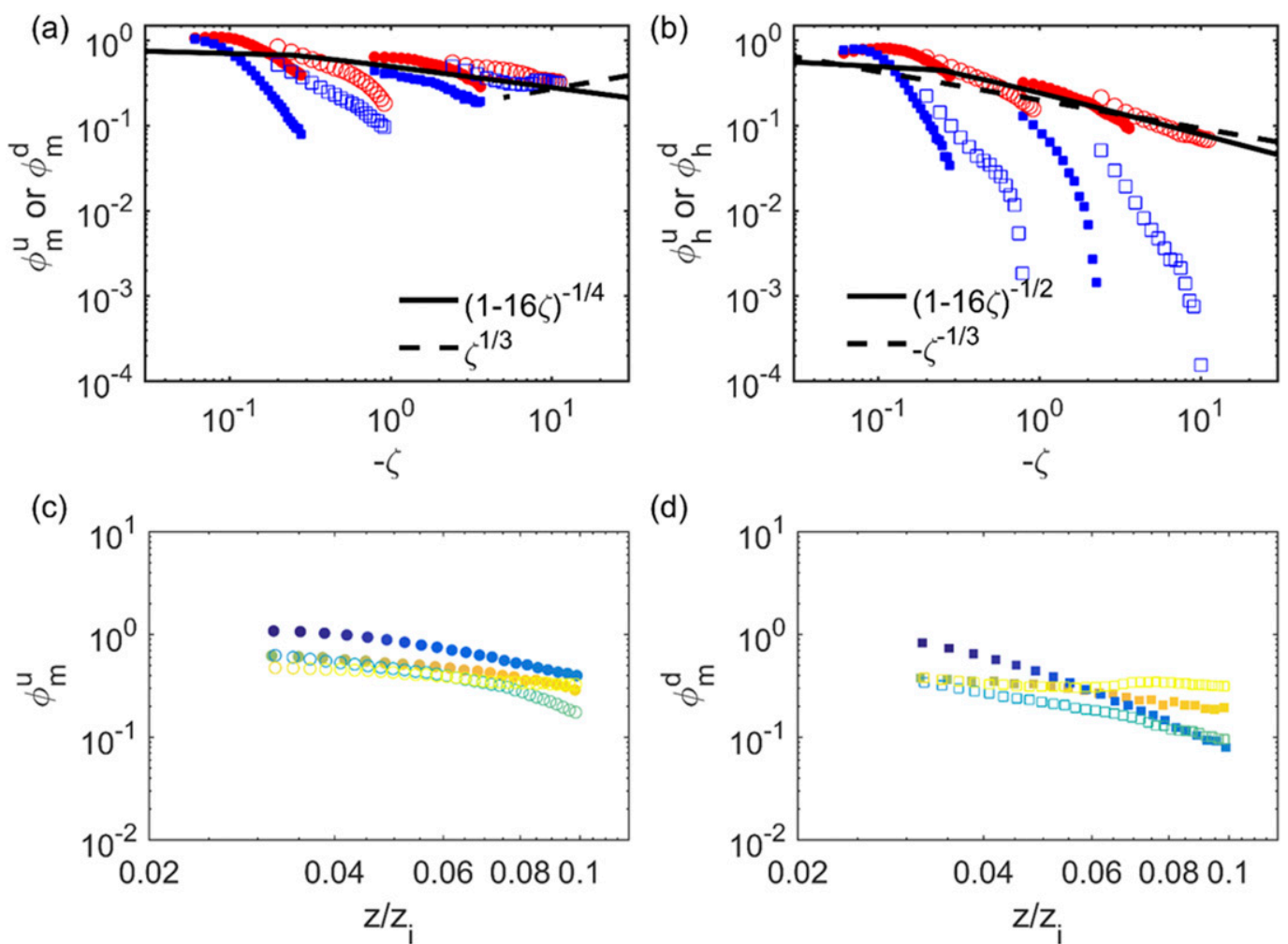

(d)
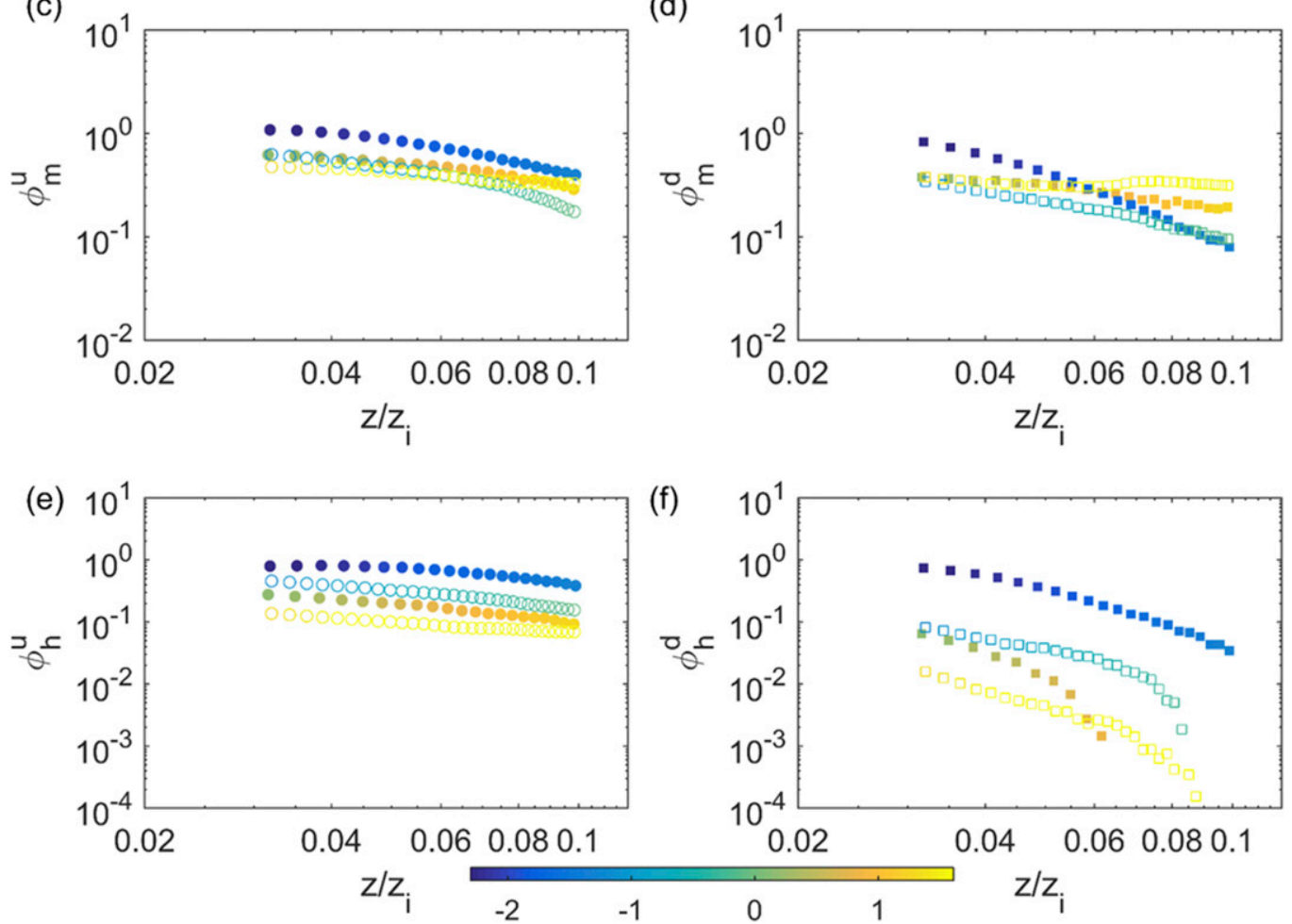

FIG. 4. Conditional $\phi_{m}$ and $\phi_{h}$, from low to high $\zeta$, the four runs correspond to LES low $B_{0}$, DNS low $B_{0}$, LES high $B_{0}$, and DNS high $B_{0}$. Red circle: $\phi_{m}^{u}$ or $\phi_{h}^{u}$; blue square: $\phi_{m}^{d}$ or $\phi_{h}^{d}$. (a) $\phi_{m}^{u}$ or $\phi_{m}^{d}$ vs $-\zeta=-z / L$, (b) $\phi_{h}^{u}$ or $\phi_{h}^{d}$ vs $-\zeta=-z / L$, (c) $\phi_{m}^{u}$ vs $z / z_{i}$, (d) $\phi_{m}^{d}$ vs $z / z_{i}$, (e) $\phi_{h}^{u}$ vs $z / z_{i}$, and (f) $\phi_{h}^{d}$ vs $z / z_{i}$. The color scale in (c)-(f) indicates $\log (-\zeta)$.

to explain the interactions between inner and outer layers such that the inactive eddies play a dynamically important role in contributing to the turbulent stress. Although we cannot explicitly demonstrate this mechanism, the similarity between $\phi_{m}^{u}$ and $\phi_{m}^{d}$ (more than that between $\phi_{h}^{u}$ and $\phi_{h}^{d}$ ) is a strong indication of interactions across scales.

However, one should be cautious about two points. First, it is worth emphasizing that MOST (with the assumption of Townsend's hypothesis of wall-attached eddies) is a dimensional analysis. Dimensional analysis, in general, does not automatically prevent interactions across scales. Second, it is important to recognize that failure to adhere to the conditions hypothesized by the dimensional reasoning means that the theory has been applied in a regime outside its applicability. Therefore, if the outer-layer effects are also important, then $z_{i}$ must also be added to the list of parameters by a similar line of reasoning to Yaglom's (1979). In addition, this raises another possibility: the use of a global $u_{*}$ value might not be a good uniform scale for both updraft and subsidence regions; could MOST be recovered using the local $u_{*}$ ? This type of reasoning about the large-scale motions modulating the small-scale structures is an active topic of 
(a)

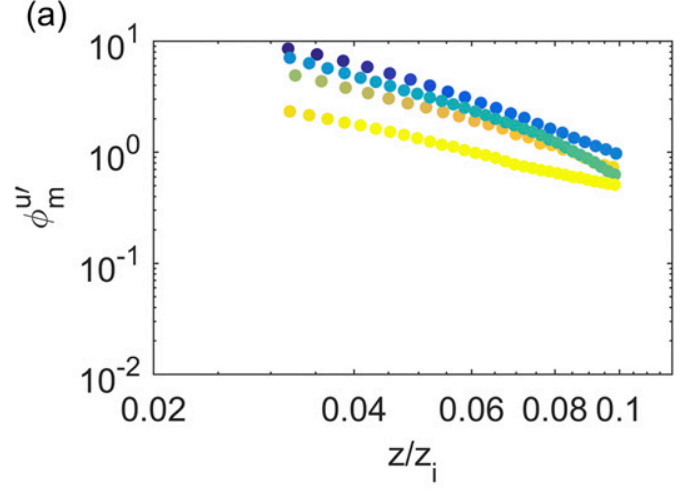

(c)

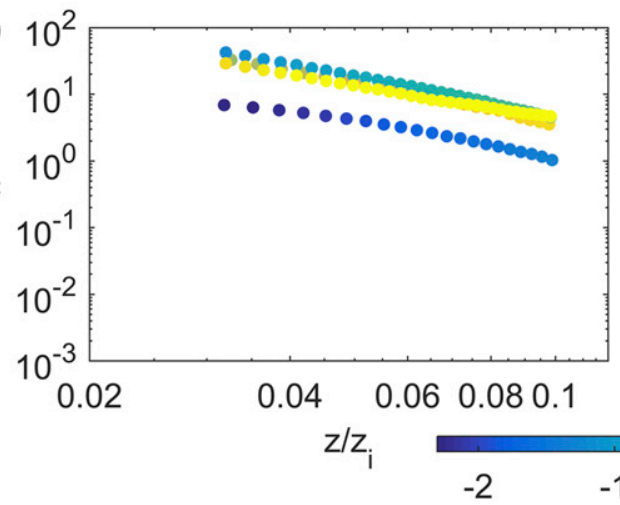

(b)

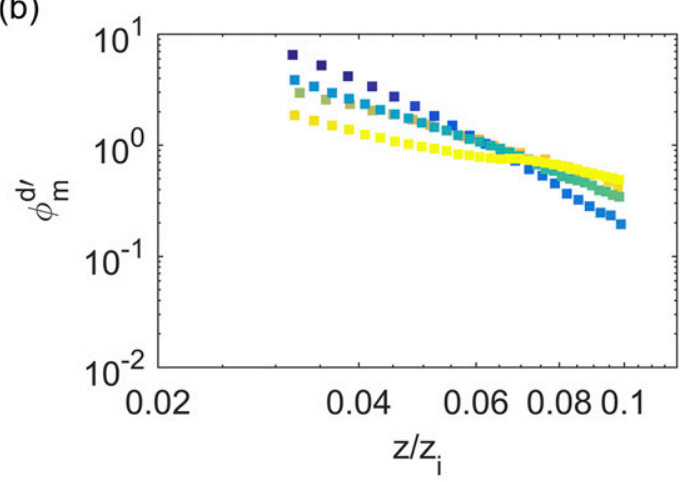

(d)

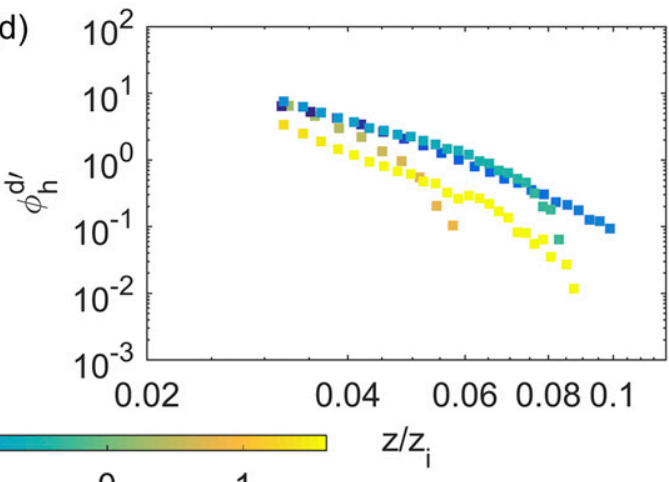

FIG. 5. As in Figs. 4c-f, but the conditional $\phi$ is normalized with $\left\{z_{i}, w_{*}, B_{0} / w_{*}\right\}$.

research (e.g., Marusic et al. 2010; Mathis et al. 2011; Baars et al. 2017). This latter possibility is tested, but the scaling with local $u_{*}$ does not collapse the updrafts and subsidence components of $\phi_{m}$ (results not shown here). Overall, we find that for momentum $\phi_{m}^{u}$ and $\phi_{m}^{d}$ exhibit similar dependence on $z / L$ and $z / z_{i}$ but oppositely for temperature; $\phi_{h}^{u}$ is correctly predicted by MOST function and thus the use of $z / L$ only.

Rather than the "indirect" influence of $z_{i}$ on the surface layer, shown in Khanna and Brasseur (1997), the direct influence of $z_{i}$ can be demonstrated by evaluating the scaling of variables with the mixed-layer scaling. We denote the dimensionless gradients scaled by the mixedlayer variables $\left(z_{i}, w_{*}\right.$, and $\left.-\overline{\theta^{\prime} w^{\prime}} / w_{*}\right)$ as $\phi_{m}^{u^{\prime}}, \phi_{m}^{d^{\prime}}, \phi_{h}^{u^{\prime}}$, and $\phi_{h}^{d^{\prime}}$. The $\phi_{h}^{d^{\prime}}$ collapses much better in Fig. 5d than in Figs. $4 \mathrm{~b}$ or $4 \mathrm{f} ; \phi_{h}^{d}$ can have extremely small $\left(\sim 10^{-4}\right)$ values compared to $\phi_{h}^{d^{\prime}}$, which is around order 1 . On the other hand, the MOST-based variables are more appropriate for the subsidence component of the horizontal momentum since $\phi_{m}^{d^{\prime}}$ deviate significantly from order one. The dissimilarity between the dimensionless gradients of $u$ and $\theta$ when plotted with different scaling regimes indicates some fundamental differences in how the horizontal momentum and temperature are transported near the surface. Section $3 \mathrm{~d}$ will explore possible reasons for this relating to turbulent coherent structures.

\section{c. Probability density function: Contributions of updrafts and subsidence}

Since the dimensionless concentration of passive scalar $s$ released into the domain follows a heightdependent initial mean profile [i.e., $s\left(z, t=t_{0}\right)=z / z_{i 0}$ ], its concentration distribution at a given height $z$ gives information about the origins of the air arriving in $s$. By tracking the distribution of $s$, we can compute the socalled transilient matrix with matrix coefficients $c_{i j}$, which represent the fraction of air in position $i$ that came from source level $j$ within some discrete time step $\Delta t$ (Stull 1993). The transilient matrix satisfies the conservation of mass; that is, $\sum_{\text {all sources } j} c_{i j}=1$. More details are given in Stull (1984). Thus, the transilient matrix indicates the origins of updrafts and downdrafts that contribute to the transport of momentum and temperature at a given height. In other words, we can quantify the nonlocal contribution to the observed flux. Physically, the transilient matrix represents the vertical mixing effect across scales. For a given reference height, the row vector of the transilient matrix represents the mixing contribution due to air from different heights.

Following the convention of Stull (1988, p. 227), the horizontal axis is the source height, while the vertical 

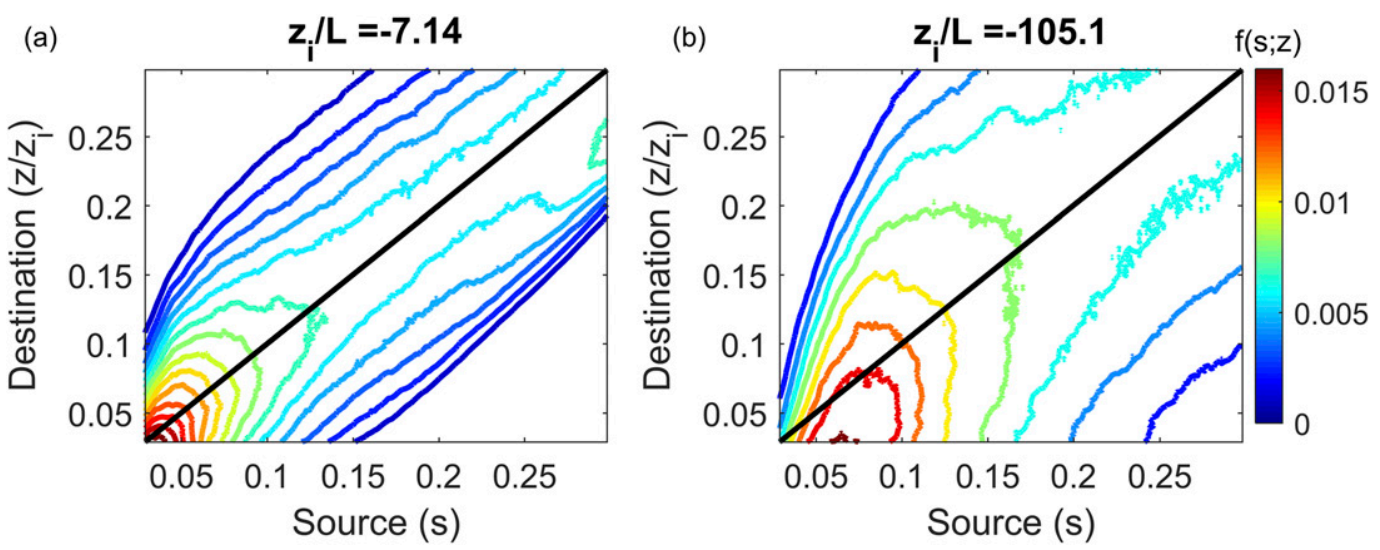

FIG. 6. The transilient matrix based on passive tracer $s$ for (a) DNS low $B_{0}$ and (b) DNS high $B_{0}$. Color scales indicate the probability density function $f(s ; z)$. Only parts of the domain above the viscous sublayer are considered here.

direction is the height of analysis. The constraint for the pdf that $\int_{\text {all } s} f(s ; z) d s=1$ is analogous to the constraint in the discretized version of the transilient matrix that $\sum_{\text {all sources } j} c_{i j}=1$. Note that $f(s ; z)$ is a function of continuous variables (i.e., the destination $z$ and the source $s$ ), whereas the transilient matrix represents mixing across discretized finite distances. In practice, since the domain is discretized and countable bins of $s$ are chosen, $c_{i j}$ is equivalent to $\int_{s}^{s+\Delta s} f(s ; z) d s$, where $\Delta s$ is the bin size.

As defined in section $2 \mathrm{c}$, the pdf $f(s ; z)$ is shown in Fig. 6 for DNS simulations at low (Fig. 6a) and high $B_{0}$ (Fig. 6b). Note that $f(s ; z)$ changes with time and depends (weakly) on the relaxation time scale. For the same $\tau$, we selected $f(s ; z)$ run at the end of the total time period of $z_{i} / 3 w_{*}$. We only consider the part of the domain where the diffusive heat flux is less than $5 \%$ of the total flux; the latter corresponds to the lower $3 \%$ of $z_{i}$ (Fig. 6). The upper-left (lower right) portion of Fig. 6 with respect to the one-to-one line indicates updraft (subsidence) because, given the particular relaxation time scale and at any level $z, s\left(x, y, z, t=t_{0}+z_{i} / 3 w_{*}\right)$ indicates where the passive tracer originates from.

If eddy diffusion (K theory) is responsible for most of the mixing process (i.e., local mixing dominates), then we expect the distribution to be symmetric around the one-to-one line and only spread around a narrow diagonal envelope, such as observed for the low-buoyancy case (Fig. 6a). This means that only eddies near the level of interest contribute to the transport. However, nonlocal mixing is reflected as a strong deviation from the one-to-one line toward the $y$ axis denoted as "destination," such as for DNS high $B_{0}$, the highly convective case (Fig. 6b). In the low-buoyancy case, we observed nearly symmetric behaviors mostly localized around the 1:1 line (Fig. 6a). On the other hand, updrafts originating from the lower surface layer are responsible for most of the surface-layer transport in the stronger buoyancy case (Fig. 6b). These updrafts are compensated by larger-scale subsidence, which originate from levels above the surface layer.

The pdf-weighted averages [i.e., Eq. (12)] of momentum and temperature fluxes can be used to quantify the contributions of updrafts and subsidence to the total fluxes. Definitions of updrafts and downdrafts are typically based on the sign of the vertical component of velocity, such as (Schumann and Moeng 1991a,b; Young 1988b,a; Zhuang 1995). We compared our analysis with those in Schumann and Moeng (1991a) by computing the "flux velocities" $\omega^{*}$ and $\omega^{* *}$ defined as $\omega^{*}=\overline{w^{\prime} f^{\prime}} /(f-\bar{f})$ and $\omega^{* *}=\overline{w^{\prime} f^{\prime}} /\left(f_{u}-f_{d}\right)$, where $f$ represents either $u$ or $\theta$; subscripts $u$ and $d$ represent the conditional average based on either the sign of $w$ (as in their analysis) or the sign of $s^{\prime}$. Similar results of the $\omega^{*}$ and $\omega^{* *}$ can be obtained using the sign of the passive tracer or the sign of $w$ compared to Schumann and Moeng (1991a; results not shown here). The pdf of $s$ is not perfect since $s$ depends on the relaxation time scale; nevertheless, it indicates contributions from different heights, which is a viable method to probe the details of nonlocal transport in the surface.

To study the impact of different air sources on the fluxes, we compute $\left\langle u^{\prime} w^{\prime} \mid s ; z\right\rangle_{0}$ and $\left\langle\theta^{\prime} w^{\prime} \mid s ; z\right\rangle_{0}$ according to Eq. (11) in Fig. 7. The asymmetry across the one-toone line reflects the unequal contributions from updrafts and subsidence. The $\left\langle u^{\prime} w^{\prime} \mid s ; z\right\rangle_{0}$ and $\left\langle\theta^{\prime} w^{\prime} \mid s ; z\right\rangle_{0}$ resemble one another in the low-buoyancy case in Fig. 7. Both are relatively symmetric around the one-to-one line, but the extreme values can be found on sources where $z / z_{i}<$ $0.15 \mathrm{~s}$. Most of the air parcels arriving at a given height in the surface layer originate from nearby levels as 

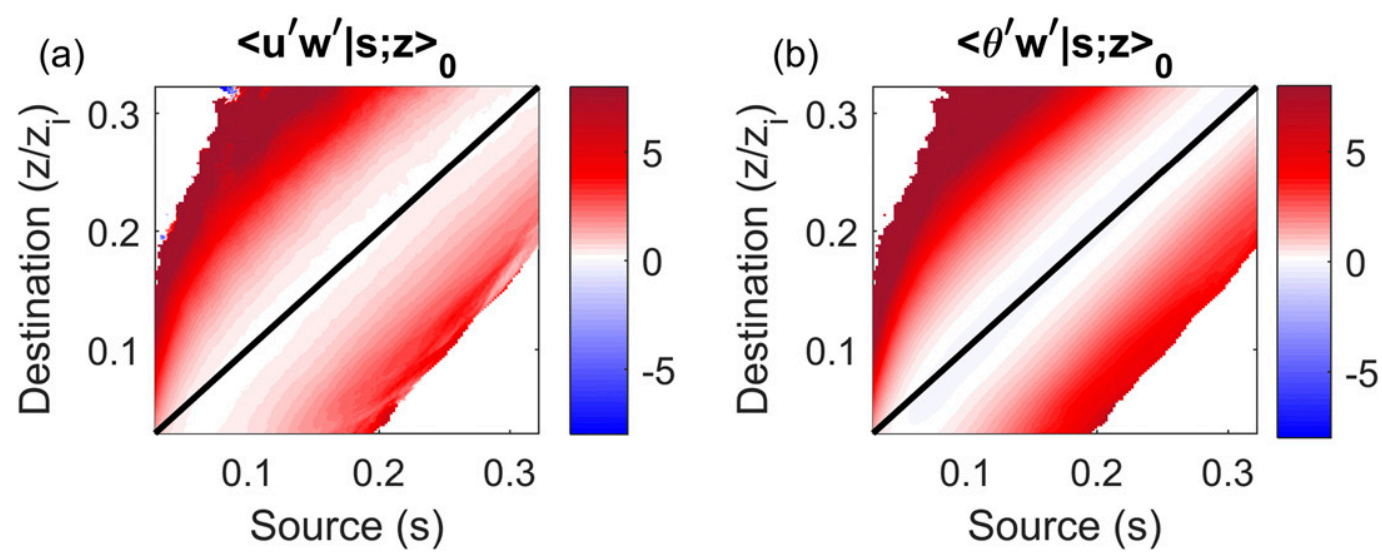

FIG. 7. Plots of (a) $\left\langle u^{\prime} w^{\prime} \mid s ; z\right\rangle_{0}$ and (b) $\left\langle\theta^{\prime} w^{\prime} \mid s ; z\right\rangle_{0}$ for the low-buoyancy DNS run at $z_{i} / L=-7.14$. The solid black line indicates the one-to-one line.

indicated by higher values of $f(s, z)$ being closer to the one-to-one line shown by the contours in Fig. 6. However, the flux contribution of this air parcel is very small since it only brings small anomalies compared to the reference level. Thus, overall, the main contribution on $\left\langle u^{\prime} w^{\prime} \mid s ; z\right\rangle_{0}$ and $\left\langle\theta^{\prime} w^{\prime} \mid s ; z\right\rangle_{0}$ is due to updrafts rising from the near surface and subsidence coming from a larger distance.

For the highly convective case (Fig. 8), the air parcels that originate from lower levels reach much higher destinations compared to the lower-buoyancy case (Fig. 7). In contrast to the shear case, negative values of $\left\langle u^{\prime} w^{\prime} \mid s ; z\right\rangle_{0}$ are observed in the subsidence section. Both positive contributions to $\left\langle\theta^{\prime} w^{\prime} \mid s ; z\right\rangle_{0}$ are due to the updraft and subsidence. These results are consistent with the results using the expected value of fluxes conditioned on $w$ in Khanna and Brasseur (1998). According to Khanna and Brasseur (1998), for both highly convective $\left(z_{i} / L=-730\right)$ and less convective $\left(z_{i} / L=-8\right)$ conditions, there is a higher contribution to momentum and temperature fluxes from the upward motion compared to the downward one. Our results also support the methodology adopted in Siebesma et al. (2007). In their study, a convective mass flux was used to represent the role of strong organized nonlocal updrafts, while the background turbulent fluxes were parameterized by the eddy-diffusivity method. The following section of this paper will further explain the observed differences between $\left\langle u^{\prime} w^{\prime} \mid s ; z\right\rangle_{0}$ and $\left\langle\theta^{\prime} w^{\prime} \mid s ; z\right\rangle_{0}$ and the effect of increasing convective instability.

Next, according to Eq. (12), we compute $\left\langle u^{\prime} w^{\prime} \mid s^{\prime} ; z\right\rangle_{N}$ and $\left\langle\theta^{\prime} w^{\prime} \mid s^{\prime} ; z\right\rangle_{N}$, which explicitly relate the contributions from a certain location to the total turbulent fluxes due to updrafts and subsidence. There are contributions to both momentum and temperature fluxes from updrafts (i.e., $s^{\prime}<0$ ) and subsidence (i.e., $s^{\prime}>0$ ), but the updraft contributions dominate transport, as shown in Figs. 9 and 10. The transport by subsidence is nonetheless far from negligible (Figs. 9a,b). The double-peak features in Figs. 9 and 10 are due to both the magnitude of the fluxes

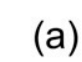

(a)

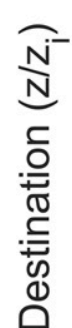

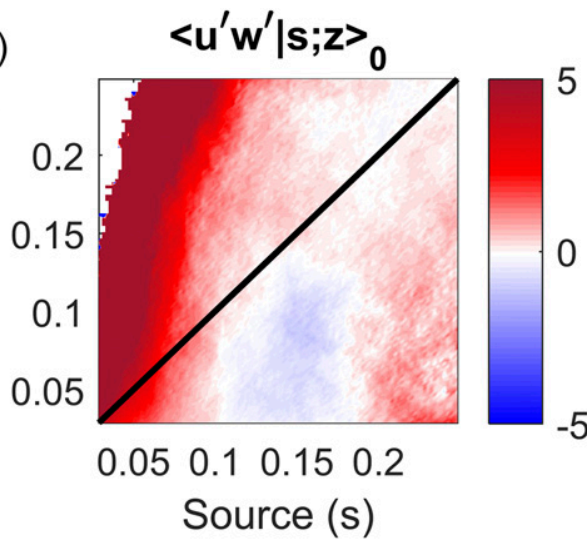

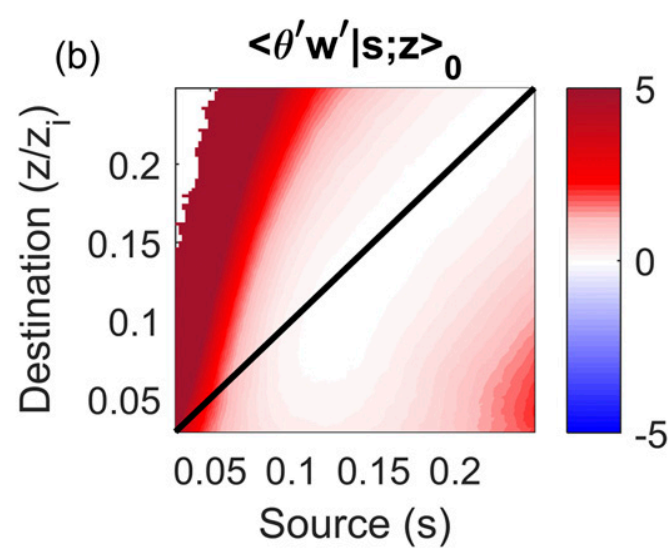

FIG. 8. As in Fig. 7, but for DNS high- $B_{0}$ run at $z_{i} / L=-105.1$. 
(a)

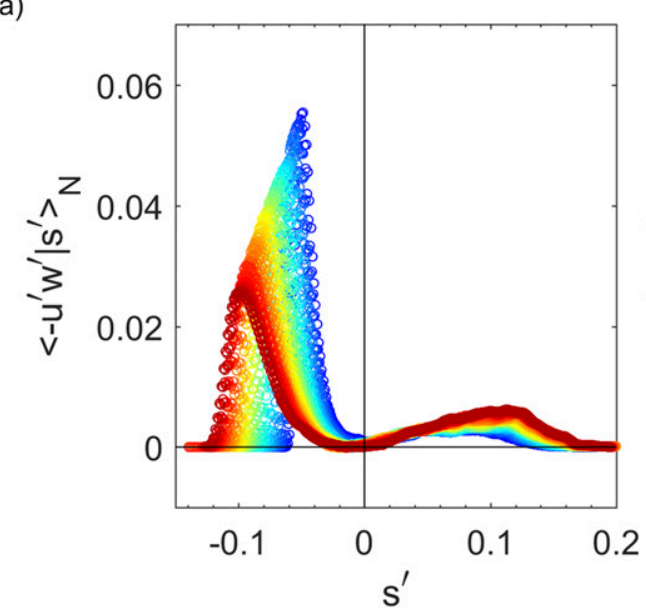

(b)

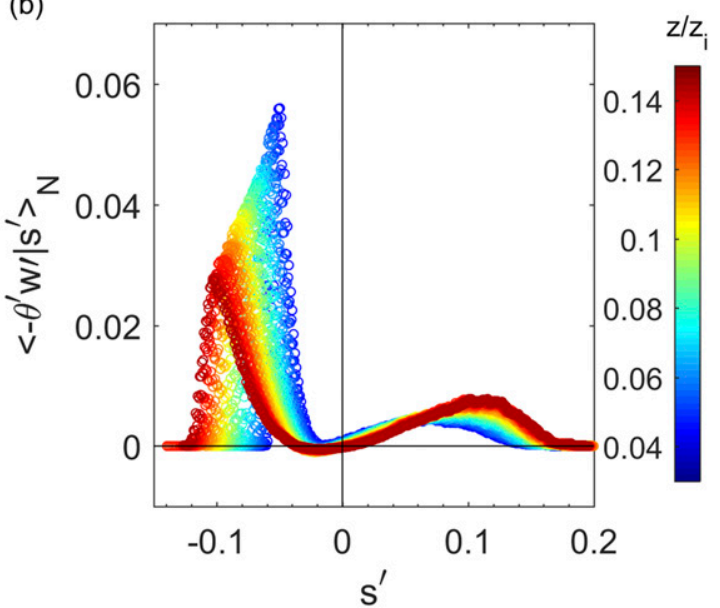

FIG. 9. The normalized flux contributions for the low-buoyancy DNS run (a) $\left\langle u^{\prime} w^{\prime} \mid s^{\prime} ; z\right\rangle_{N}$ and (b) $\left\langle\theta^{\prime} w^{\prime} \mid s^{\prime} ; z\right\rangle_{N}$.

from a given origin and their relative abundance measured by $f\left(s^{\prime} ; z\right)$. Despite the similarities in terms of the double-peak features, one distinction between the lowand high- $B_{0}$ runs is the difference in subsidence contribution to the momentum flux and the nonnegligible negative subsidence contributions, shown in Fig. 8a. This nontrivial difference between momentum and temperature fluxes in the high- $B_{0}$ run will be further examined in the next section. Another difference between Fig. 9 and Fig. 10 is that the peaks in Figs. 10a and $10 \mathrm{~b}$, which indicate the updraft contributions to $\left\langle u^{\prime} w^{\prime} \mid s^{\prime} ; z\right\rangle_{N}$ and $\left\langle\theta^{\prime} w^{\prime} \mid s^{\prime} ; z\right\rangle_{N}$, are broader than the lowbuoyancy case for $z<0.1 z_{i}$. This means that a single length scale based on wall distance (modified by MOST scaling) could be an oversimplification. To further illustrate this point, we show the fraction of momentum and temperature fluxes conditioned on updrafts $\left(\left\langle u^{\prime} w^{\prime} \mid s^{\prime} \leq 0 ; z\right\rangle /\left\langle u^{\prime} w^{\prime}\right\rangle\right.$ and $\left.\left\langle\theta^{\prime} w^{\prime} \mid s^{\prime} \leq 0 ; z\right\rangle /\left\langle\theta^{\prime} w^{\prime}\right\rangle\right)$ and subsidence $\left(\left\langle u^{\prime} w^{\prime} \mid s^{\prime}\right\rangle 0 ; z\right\rangle\left\langle\left\langle u^{\prime} w^{\prime}\right\rangle\right.$ and $\left.\left.\left\langle\theta^{\prime} w^{\prime} \mid s^{\prime}\right\rangle 0 ; z\right\rangle /\left\langle\theta^{\prime} w^{\prime}\right\rangle\right)$ in Figs. 11a and 11c. In the low-buoyancy run, the subsidence contributions account for approximately $25 \%$ of the total momentum fluxes at around $z / z_{i}=0.03$, and the fraction increases slightly with height (Fig. 11a). In the high- $B_{0}$ run, the fractional contribution of momentum flux in subsidence is close to zero because of the cancellation of fluxes of both signs. We hypothesize that one can interpret the peak values of $s^{\prime}$ as indication of the dominant length scale that contributes to momentum and temperature fluxes. Note that we analyzed the pdfs after $f(s ; z)$ becomes quasi steady after about the first $20 \%$ of the total duration of the tracer experiment. Only results from instantaneous pdfs at the end of the tracer experiment are shown here because we are interested in relating the instantaneous (a)

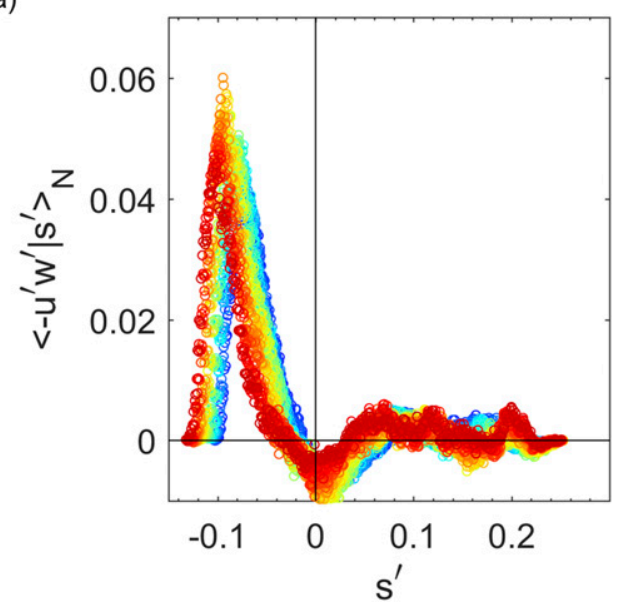

(b)

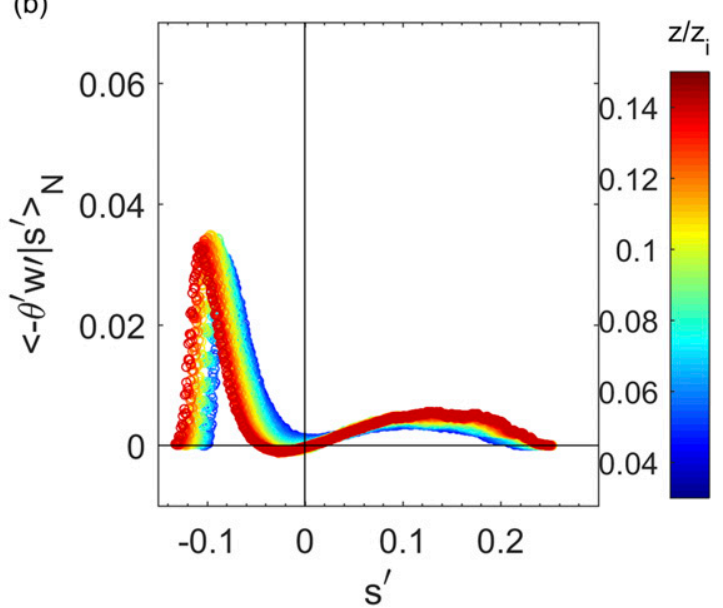

FIG. 10. As in Fig. 9, but for the high- $B_{0}$ run. 


\section{Low buoyancy}

(a)

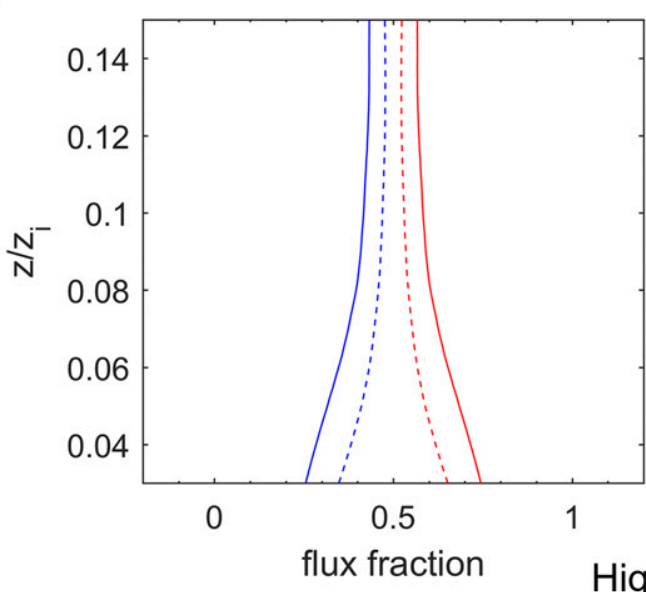

(c)

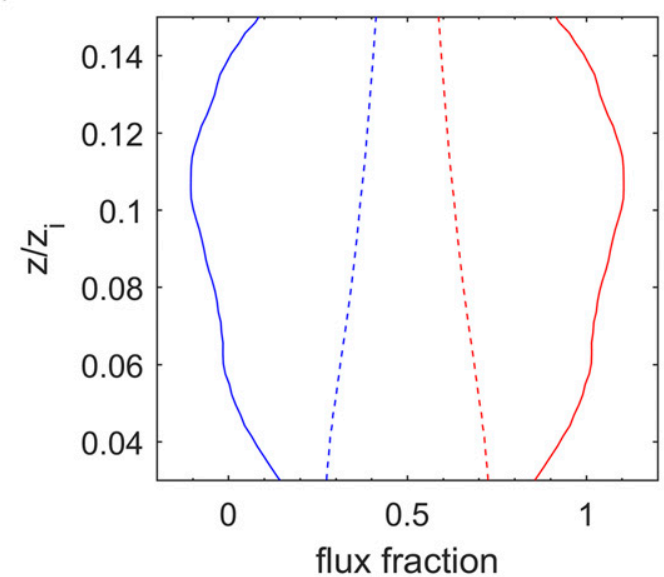

(b)

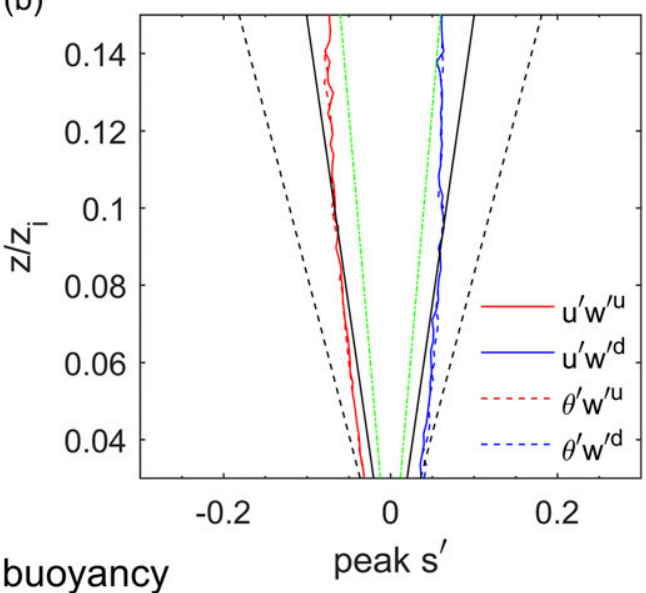

(d)

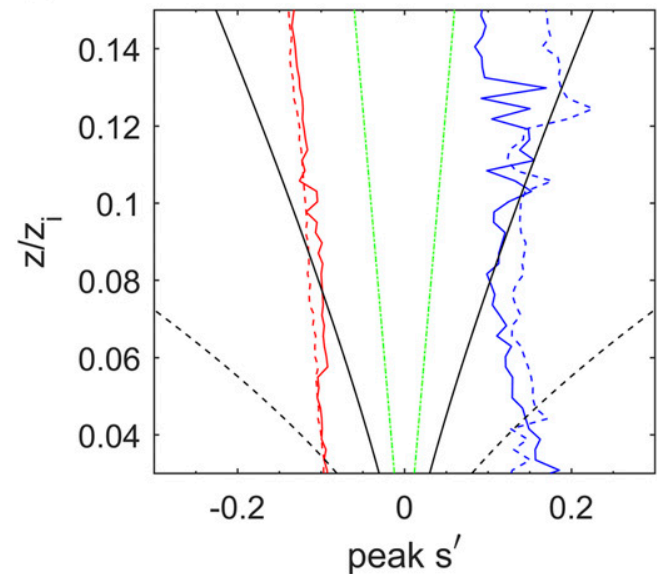

FIG. 11. Fraction of momentum and temperature fluxes conditioned on updrafts $\left(\left\langle u^{\prime} w^{\prime} \mid s^{\prime} \leq 0 ; z\right\rangle /\left\langle u^{\prime} w^{\prime}\right\rangle\right.$ and $\left.\left\langle\theta^{\prime} w^{\prime} \mid s^{\prime} \leq 0 ; z\right\rangle /\left\langle\theta^{\prime} w^{\prime}\right\rangle\right)$ and subsidence $\left(\left\langle u^{\prime} w^{\prime} \mid s^{\prime}>0 ; z\right\rangle /\left\langle u^{\prime} w^{\prime}\right\rangle\right.$ and $\left.\left\langle\theta^{\prime} w^{\prime} \mid s^{\prime}>0 ; z\right\rangle /\left\langle\theta^{\prime} w^{\prime}\right\rangle\right)$ for (a) low- $B_{0}$ run and (c) high- $B_{0}$ run; peak s' at which the maximum of $\left\langle u^{\prime} w^{\prime} \mid s^{\prime} ; z\right\rangle_{N}$ or $\left\langle\theta^{\prime} w^{\prime} \mid s^{\prime} ; z\right\rangle_{N}$ occurs for $s^{\prime}<0$ (updraft) or $s^{\prime}>0$ (subsidence) for (b) low- $B_{0}$ run and (d) high- $B_{0}$ run. Green dash-dotted line: $\kappa z / z_{i}$; black solid line: $\kappa z / \phi_{m} z_{i}$; black dotted line: $\kappa z / \phi_{h} z_{i}$.

coherent structures to the pdf of $s$. The variability of $s_{\text {peak }}^{\prime}$ defined as the standard deviation of $s_{\text {peak }}^{\prime}$ divided by the time-averaged values of $s_{\text {peak }}^{\prime}$ is between $2 \%$ (updrafts) to at most $15 \%$ (subsidence). Details of its variability are shown in appendix A. The green dashdotted lines in Figs. $11 \mathrm{~b}$ and $11 \mathrm{~d}$ correspond to the estimates of $s_{\text {peak }}^{\prime}$, where maxima of $\left\langle u^{\prime} w^{\prime} \mid s^{\prime} ; z\right\rangle_{N}$ and $\left\langle\theta^{\prime} w^{\prime} \mid s^{\prime} ; z\right\rangle_{N}$ occur, given by $\left|s_{\text {peak }}^{\prime}(z)\right|=\kappa z / z_{i}(\kappa=0.40$ being the von Kármán constant). The mixing-length $l_{m(h)} / z_{i}$ based on MOST (Businger et al. 1971; McPhee 1994) are shown for comparison in Figs. 11b and 11d, where $l_{m}=\kappa z / \phi_{m}(\zeta)$ (black solid line) and $l_{h}=\kappa z / \phi_{h}(\zeta)$ (black dotted line) and $\phi_{m}, \phi_{h}$ are computed from simulations; $l_{m}, l_{h}$, and $s_{\text {peak }}^{\prime}$ capture the vertical variation of the dominant length scale for the low- $B_{0}$ case quite well for both updrafts and subsidence. For the case with high $B_{0}$, however, our hypothesis about the dominant length scale is incompatible with the mixinglength model. Furthermore, the dominant length scale for subsidence does not scale with $z$ at all, although arguably, there is not a well-defined peak for subsidence in this case. It is interesting to note that $\left|s_{\text {peak }}^{\prime}(z)\right|=f\left(z_{i} / L\right) \kappa z / z_{i}$ could potentially be a model of the mixing length for the updraft since $\left|s_{\text {peak }}^{\prime}(z)\right|=\kappa z / z_{i}$ is parallel to the dominant length scale for both runs. We compared the results for corresponding LES runs, and similar conclusions can be reached (see figures in appendix B).

One important conclusion from the analysis in this section is that, close to the wall, the outer-region 
(a)

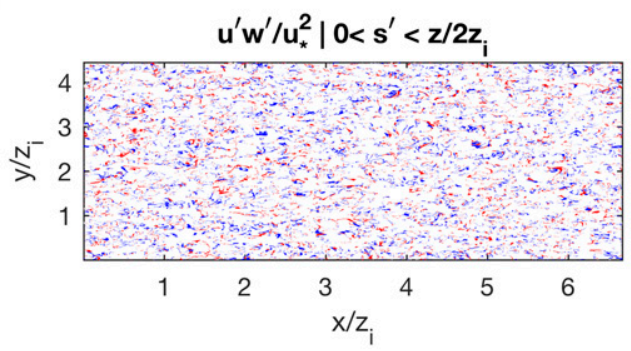

(c)

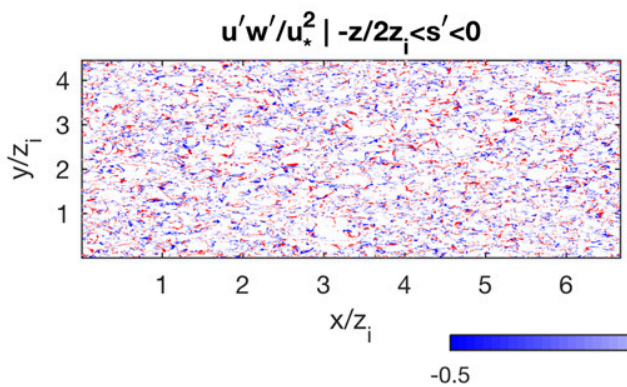

(b)

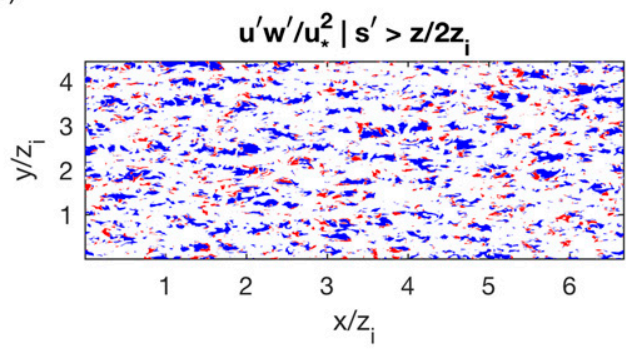

(d)

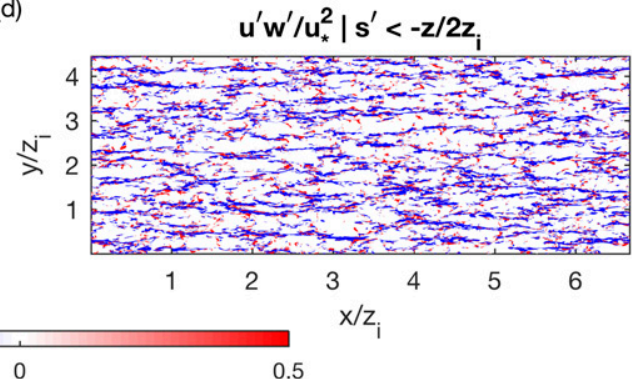

FIG. 12. The low-buoyancy DNS run: $x-y$ cross sections of $u^{\prime} w^{\prime}$ at $z / z_{i}=0.08$ conditioned on $s^{\prime}$, where $s^{\prime}<0(>0)$ represents updrafts (subsidence).

contribution to both momentum and temperature fluxes is far from being negligible. In fact, for temperature, it is of the same order of magnitude as the updraft contribution. Referring back to the distinct stability slopes between $\phi_{h}^{u}$ and $\phi_{h}^{d}$ in Fig. $4 \mathrm{~b}$, it reinforces the idea that the eddy-diffusivity-based method using the inner scales is invalid. Moreover, our results demonstrate that the contributions due to subsidence should be explicitly accounted for.

\section{d. Analyses of coherent structures}

Figure 12 and Fig. 13 show the instantaneous $x-y$ cross sections of momentum and temperature fluxes from DNS at $z=0.08 z_{i}$ for low- and high- $B_{0}$ cases, respectively, conditioned on different criteria based on $s^{\prime}$. With higher instability, the updrafts change from elongated streaks to cellular structures, as expected. The general trend of transition from rolls to cellular structures with increasing buoyancy has been well documented in previous studies (e.g., Moeng and Sullivan 1994; Salesky et al. 2017). Here, we focus on the comparisons between $u^{\prime} w^{\prime}$ and $\theta^{\prime} w^{\prime}$. The turbulent fluxes are qualitatively similar in the low-buoyancy case, in which $u^{\prime}$ is highly correlated with $\theta^{\prime}$ in both updrafts and downdrafts. The elongated streaks of high correlation between negative $u^{\prime}$ and positive $\theta^{\prime}$ form the "sheetlike" updrafts, which are surrounded by the "broader regions" of subsidence, similar to findings in Khanna and Brasseur (1998). The shear-induced low-speed streaks and the coherent sheets of upward-moving fluid are the main contributors to $u^{\prime} w^{\prime}$ and $\theta^{\prime} w^{\prime}$ in the surface layer. The flux contribution for $u^{\prime} w^{\prime}$ given $s^{\prime}<-z / 2 z_{i}$ (i.e., air parcels coming upward from a distance farther than $z / 2 z_{i}$ ) shown in Fig. $12 \mathrm{~d}$ is $69 \%$ of the total flux, and the contribution is $63 \%$ for $\theta^{\prime} w^{\prime}$ (not shown here). The broader regions in subsidence originate from levels higher than $z / z_{i}$, and they are surrounded by regions of updrafts. These broader regions account for a majority of the fluxes due to subsidence. For example, they account for $24 \%$ (35\%) of $u^{\prime} w^{\prime}\left(\theta^{\prime} w^{\prime}\right)$ given $s^{\prime}>z / 2 z_{i}$. The updraft conditioned on $s^{\prime}<-z / 2 z_{i}$ contributes to a higher fraction of momentum and temperature flux compared to its counterpart in subsidence.

In general, transport asymmetry has been thoroughly discussed in the context of top-down and bottom-up diffusion (Moeng and Wyngaard 1984, 1989; Wyngaard and Brost 1984; Wyngaard and Weil 1991; Wyngaard and Moeng 1992) for levels above the surface layer. The transport asymmetry has also been used to account for the nonlocal effects, such as the closure for nonlocal heat fluxes, for example, in Ghannam et al. (2017). With the passive tracer, we show that the transport asymmetry present in the surface layer is related to the flux imbalance in the coherent sheets of thermals and those broader regions between the thermals. Recent work by Ghannam et al. (2017) has revealed that the eddy-diffusivity mass-flux modeling approach (e.g., Siebesma et al. 2007) and the relaxed eddy accumulation 
(a)

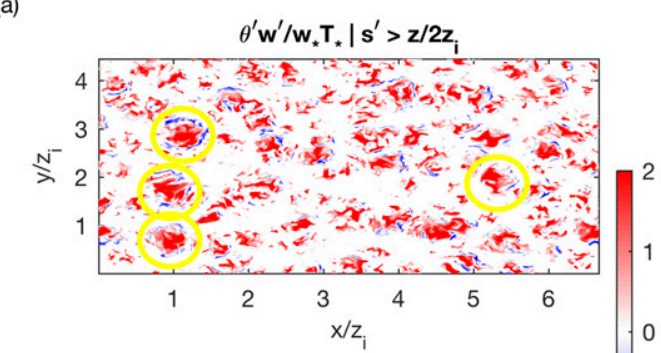

(c)

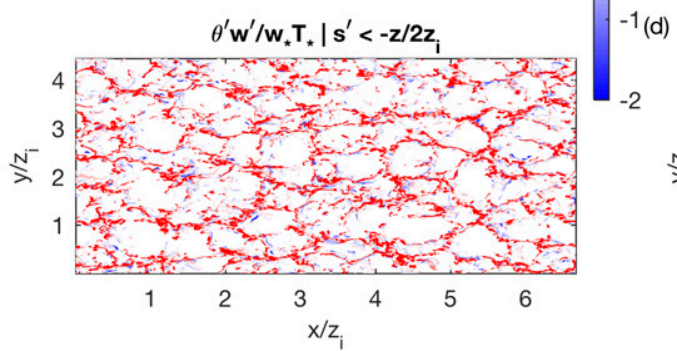

(b)

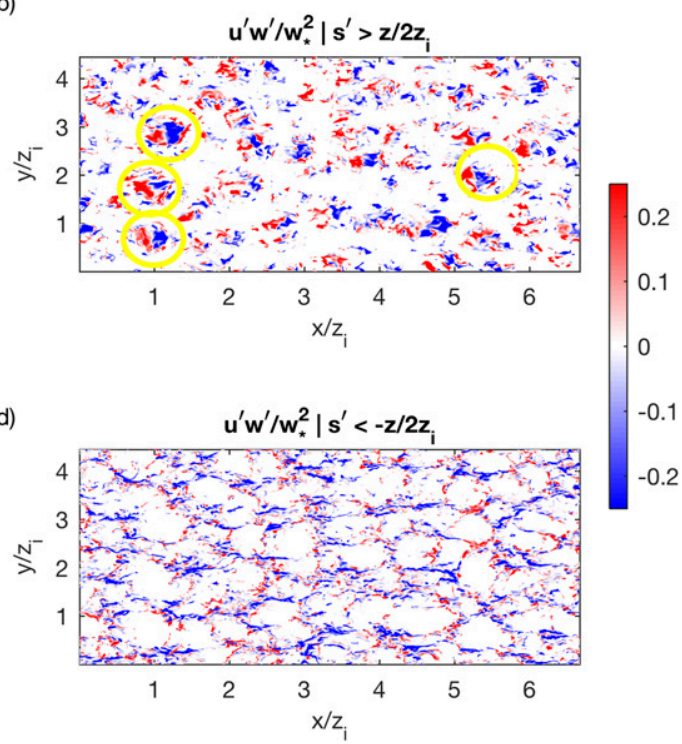

FIG. 13. As in Fig. 12, but for the case of high- $B_{0}$ DNS run. Yellow circles correspond to structures in subsidence where $u^{\prime} w^{\prime}$ and $\theta^{\prime} w^{\prime}$ are different.

method (e.g., Businger and Oncley 1990; Katul 1994) implicitly assume symmetry in transport due to updraft and subsidence. However, these methods can potentially be biased since they do not account for the transport asymmetry due to the large-scale coherent structures. Furthermore, no eddy-diffusivity mass-flux modeling approach explicitly represents the impact of downdraft or subsidence in the surface layer. Given that the $\phi_{h}$ conditioned on subsidence substantially deviate from the classic MOST scaling (Fig. 4b), this flux-contributing part of subsidence could be further elucidated in future studies.

The most striking feature in Fig. 13 is that some regions appear symmetric, for example, at $(x, y) / z_{i} \sim(1.1$, $0.8),(1.1,2),(1.2,3)$, and $(6,2.5)$, which are circled in Fig. 13. Momentum flux $u^{\prime} w^{\prime}$ of both signs are present in general. Such occurrence of both positive and negative regions inside the cellular subsidence is markedly different from $\theta^{\prime} w^{\prime}$, where high- $\theta^{\prime}$ regions are present in these circle regions. As proposed in Mahrt (1991), the correlation between the fluctuating horizontal velocity component and the vertical motion can show a systematic phase difference because the horizontal convergence "becomes more centered (in-phase) with respect to the thermals so $u$ and $w$ are more out-of-phase" (Mahrt 1991, p. 486). Based on experimental and numerical evidence (e.g., Wilczak 1984), the correlation between temperature fluctuations and vertical velocity fluctuations is however less affected. The spatial segregation of momentum and heat fluxes near the canopy top was also demonstrated by Patton et al. (2016) using LES with quadrant analysis, and they concluded that $u^{\prime}>0$ and $w^{\prime}<0$ typically occur in regions of sinking motions where the ABL-scale winds are in the positive $x$ direction (their Fig. 15b). Here, we highlight that large positive $u^{\prime} w^{\prime}$ also systematically occur in the same region. To our best knowledge, these large positive regions of $u^{\prime} w^{\prime}$ have not been demonstrated explicitly in previous studies. We find that the horizontal divergence (Figs. 13d and 14d) becomes more "centered" and coincides with regions of subsidence. Thus, $u^{\prime}$ of both signs are present in the subsidence region. As a result, the systematic phase difference between $u^{\prime}$ and $w$ arises in these sinking "cores" in regions of subsidence. Such countergradient momentum flux (but not the heat flux counterpart) has been reported by Wilczak (1984) by analyzing the three-dimensional structures of convective plumes. As the countergradient momentum flux becomes more dominant with increasing convective instability (Salesky et al. 2017), we show that in regions of subsidence, the outer interaction (i.e., $u^{\prime}<0 w^{\prime}<0$ ) occur concurrently with a strong sweeping event (i.e., $u^{\prime}>0 w^{\prime}<0$ ), as shown by the circled regions in Fig. 13d. According to Townsend (1961, p. 161), the inactive component of turbulence originating from the outer layer "is a meandering or swirling motion made up from attached eddies of large size" and "does not transfer momentum or interact with the universal (active) component." However, as convective instability sets in, the coherent motion in regions of subsidence does transfer momentum, although the overall transport can cancel out or even be countergradient, especially for a highly convective case. For the less unstable case shown in 
(a)

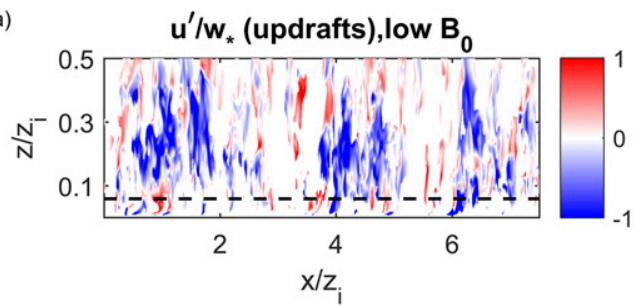

(c)

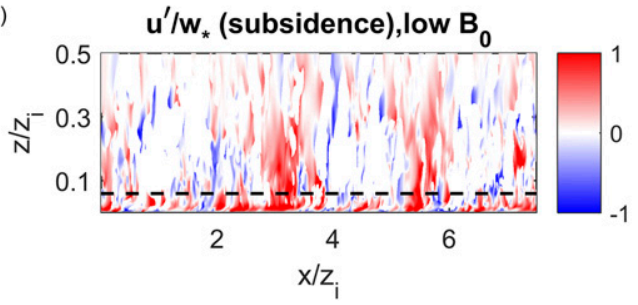

(b)

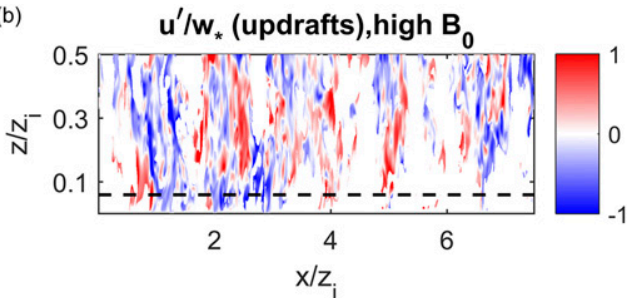

(d)

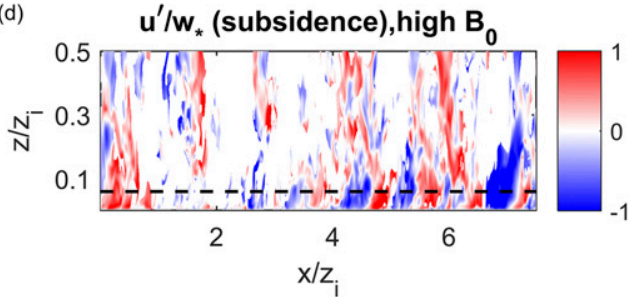

FIG. 14. The $x-z$ cross sections of $u^{\prime}$ in DNS runs: (a),(c) low- $B_{0}$ case and (b),(d) high- $B_{0}$ case. The black dotted line indicates the height where Figs. 12 and 13 are obtained.

Fig. 12b, the coherent eddies from the outer layer indeed are the second largest contribution to momentum and temperature fluxes.

To further elucidate the observed differences between the high- and low-buoyancy DNS runs and between $u^{\prime} w^{\prime}$ and $\theta^{\prime} w^{\prime}$, the $x-z$ cross sections of $u^{\prime}$ at $y=L_{y} / 2$ for both the low- and high- $B_{0}$ runs are investigated (Fig. 14); $u^{\prime}$ of negative sign is shown in Fig. 13a, and the influence of subsidence in the near-wall region is demonstrated in Fig. $14 \mathrm{c}$ by the predominant high streamwise momentum regions of red color. Thus, Fig. 14c shows that connected structures of subsidence can extend all the way down to the near surface, which is similar to findings in Zilitinkevich et al. (2006), where they used a proper orthogonal decomposition method and found that largescale eddies directly influence the near-surface region. The extension of large-scale eddies to the near-surface region is also consistent with the top-down mechanism (Hunt and Morrison 2000; Morrison 2007). In the highly convective case, it can be seen nonetheless that $u^{\prime}$ of both positive and negative values alternate in the subsidence region (e.g., along the dotted black line and at $\left.x / z_{i} \sim 4-6.5\right)$, which are signatures of the almost symmetric positive and negative $u^{\prime} w^{\prime}$ regions observed in Fig. 13d. These sinking regions of high momentum in the outer layer diverge near the wall because of mass continuity, giving rise to the alternating signs of $u^{\prime}$ that span the entire ASL.

The fluctuating $u^{\prime}$ component in regions of subsidence in a sheared heated boundary layer is often conceptualized as faster horizontal motion (Mahrt 1991) being carried in the downdrafts, which is commonly illustrated in a conceptual picture, such as Fig. 3 in McNaughton and Brunet (2002). However, based on our findings, the pattern of $u^{\prime}$ in subsidence regions is subtler than a highmagnitude $u^{\prime}$ being passively brought from aloft to the surface. Hence, we propose a different representation of the momentum compared to temperature transport in the surface layer, which further emphasizes the impact of divergence in regions of subsidence as illustrated in Fig. 15.

At higher convective instability (Fig. 15a), the updrafts rise relatively vertically, which is similar to the observations of plumes becoming more vertical with increasing $-z_{i} / L$ (Wilczak and Tillman 1980; Siebesma et al. 2007). In regions of subsidence, though, which are between the vertically rising thermals, diverging horizontal motions result in the characteristic side-by-side occurrence of positive and negative $u^{\prime}$ in Fig. 13d. Such diverging horizontal motions are related to the microfront pressure effect (Mahrt 1991), where the flow decelerates immediately behind the front (i.e., the interface between subsidence and an updraft) as the "faster gusts" descending into the surface layer. Both downgradient and countergradient momentum fluxes occur in regions of subsidence, leading to inefficiency of momentum transport. Without the microfront pressure effect and negligible effect of mixing across the interface between thermals and subsidence, $\theta^{\prime}$ is well correlated with the vertical motions. For stronger shear, the updrafts appear as streaks that are tilted in the streamwise direction (Fig. 15b), leading to the elongation of the updrafts in the direction of the mean shear. Because of the microfront pressure effect, the flow in the leading edge of an updraft is horizontally accelerated (Mahrt 1991), giving rise to reduced magnitude of negative $u^{\prime}$ in the updraft. Similarly, the flow in the trailing edge of a subsidence is horizontally accelerated, increasing the 
(a)

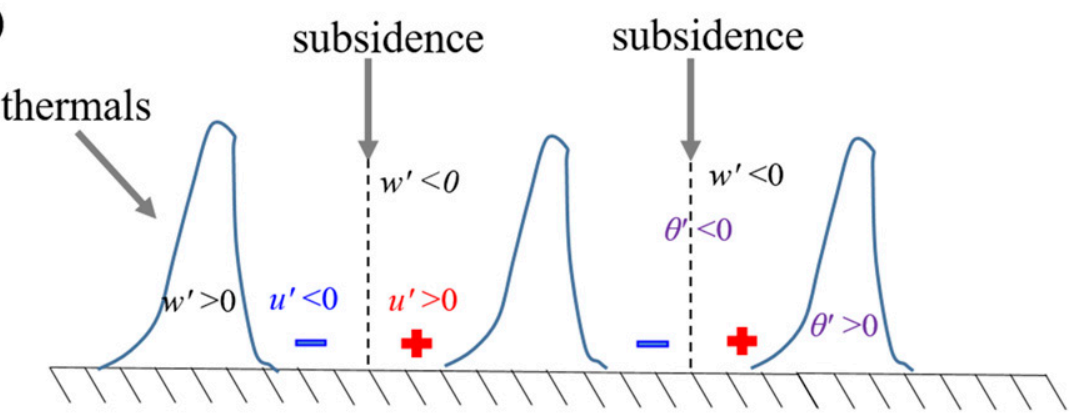

(b)

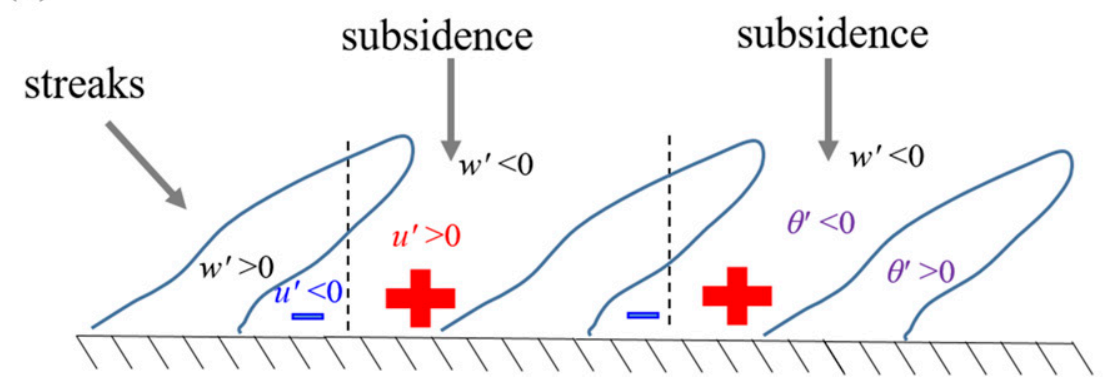

FIG. 15. Schematic diagram illustrating the change in coherent structures and the behaviors of velocity and temperature associated with these structures for (a) a highly convective case and (b) shear-dominant case. The red and blue signs represent if $u^{\prime}>0$ or if $u^{\prime}<0$; the plus sign of larger size qualitatively indicates a high magnitude of $u^{\prime}$.

magnitude of positive $u^{\prime}$. Hence, the spatial distribution of $u^{\prime}$ is asymmetric in regions of subsidence; $\theta^{\prime}$ associated with the inclined streaks can also be identified as distinct ramp structures as shown in section 2 and Fig. 1.

\section{Summary and conclusions}

LES and DNS were performed for a convective boundary layer heated from the surface and growing against a stably stratified fluid, with varying degrees of convective stability. For better comparison of LES and DNS, we carried out systematic dimensional analysis based on the LES subgrid scale and Kolmogorov microscale while matching the buoyancy Reynolds number and the Froude number. Using a passive tracer that relaxes toward a profile as an indicator of the sources of the air parcel, we show that this method successfully captures the characteristic ramp structures in temperature. This method also enables conditional sampling based on the origins of the passive tracer.

The MOST similarity functions for the mean horizontal velocity $\phi_{m}$, temperature $\phi_{h}$, variance of vertical velocity $\phi_{\sigma_{w}}$, and variance of temperature $\phi_{\sigma_{\theta}}$ are computed from both LES and DNS.

The comparisons between the computed similarity functions and the predicted ones in the literature demonstrate a larger scatter for $\phi_{m}$ compared with $\phi_{h}$, which is in agreement with previous studies such as Khanna and Brasseur (1997), Johansson et al. (2001), and Maronga and Reuder (2017). Estimates of $\phi_{m}$ and $\phi_{h}$ conditioned on updrafts or subsidence lead to two implications, which we further examine through analysis of turbulent coherent structures. First, the similarity in $\phi_{m}^{u}$ and $\phi_{m}^{d}$ suggests that inner and outer regions interact and a clear signature of the outer influence is observable in the near-surface region. Although the dependence of $\phi_{m}$ on both $z / L$ and $z / z_{i}$ is well recognized in the literature, $\phi_{m}^{u}$ and $\phi_{m}^{d}$ both demonstrate MOST-based scaling and systematic variation with $z / z_{i}$. Second, $\phi_{h}$ conditioned on subsidence does not follow the MOST scaling since it is far from being order 1 . The "nearground sweeping motions from mixed-layer eddies that scale on $z_{i}$ " (Khanna and Brasseur 1997, p. 255) are conjectured to explain the influence of $z_{i}$ on the surface layer. Figure 5d explicitly demonstrates that $\phi_{h}^{d}$ follows the mixed-layer scaling and the organized subsidence structures (shown in Figs. 12d, 13d) give rise to this direct influence of $z_{i}$ on the surface layer. The dissimilarity in $\phi_{h}^{u}$ and $\phi_{h}^{d}$ further indicates that it will be useful to have separate parameterizations for transport due to updraft and subsidence. This is especially pertinent in the eddy diffusivity mass flux (EDMF) modeling 
approach (e.g., Siebesma et al. 2007), since no current EDMF model accounts for subsidence despite its nonnegligible contribution to turbulent fluxes.

Using conditionally averaged fluxes, we demonstrate that extreme values in fluxes at $z$ arise when the air parcels originate from a large distance away from $z$. However, the maxima of $\left\langle u^{\prime} w^{\prime} \mid s^{\prime} ; z\right\rangle_{N}$ and $\left\langle\theta^{\prime} w^{\prime} \mid s^{\prime} ; z\right\rangle_{N}$ are observed for $s^{\prime}<0$ (i.e., updrafts). The dominant length scales for updrafts and subsidence are compatible with the mixing-length model in the slightly unstable case. However, it is inappropriate to use the MOSTbased mixing length (or $K$ theory) with increasing convective instability, since broadening of the peaks in $\left\langle u^{\prime} w^{\prime} \mid s^{\prime} ; z\right\rangle_{N}$ and $\left\langle\theta^{\prime} w^{\prime} \mid s^{\prime} ; z\right\rangle_{N}$ indicates that eddies of a wider range of length scales are involved.

Overall, going back to the two questions raised in the introduction regarding 1) the organized motions in the ASL and 2) the implications of their structural change, we show how the coherent structures change with increasing convective instability in the surface layer. The organized motions in the ASL are due to eddies originating from a distance up or down. They are also the main contributors to the transport asymmetry between updraft and subsidence. The structural change from "thermal sheets" to "plumes" alters how the outer-layer eddies impinge on the near-surface region, suggesting a possible effect of the "top-down" mechanism proposed in Hunt and Morrison (2000). Especially in the high- $B_{0}$ case, subsidence contributions to momentum flux differ from that for temperature flux. The additional effect of horizontal pressure leads to different behaviors in $u^{\prime}$ compared to $\theta^{\prime}$ in the organized subsidence. When vertical plumes dominate in highly convective conditions, opposite signs of $u^{\prime}$ and $\theta^{\prime}$ are observed in these subsiding downdrafts. In more unstable cases, the decreasing efficiency in the turbulent transport of $u$ compared to $\theta$ is related to this mechanism that occurs in subsidence. An alternative interpretation is that the geometric symmetry in the coherent structures increases the systematic phase lag between $u$ and $w$. Thus, the pressure-induced phase relation among different components could be the primary reason for differences between the transport of momentum versus temperature.

One of the key messages from the study is that interactions between the outer and inner layers may differ from those assumed in Townsend's attached eddy hypothesis. The coherent subsidence motions in the surface layer, particularly those from above the surface layer, actively contribute to momentum and temperature fluxes. In addition to including the outer-layer scaling parameter such as $z / z_{i}$ in the universal relations for MOST-based variables as suggested by previous studies, the subsidence component follows the mixedlayer scaling, especially for temperature. It would be of interest to incorporate the mechanism of subsidence in future studies that aim at modeling turbulent heat fluxes in the unstable surface layer.

Acknowledgments. We thank the three anonymous reviewers for their constructive feedback. P.G. acknowledges funding from the NSF CAREER Program (NSF-EAR-1552304). K.A.M. acknowledges funding from a Ziff Environmental Fellowship from Harvard University. The simulations were performed on the computing clusters of the National Center of Atmospheric Research under Project UCLB0017.

\section{APPENDIX A}

\section{Sensitivity Test of Relaxation Time Scale for Passive Tracer}

A constant relaxation term $-[s-s(t=0)] / \tau$ is added to the advection-diffusion equation of $s$, where $\tau=$ $n z_{i 0} / w_{*}$ is the relaxation time scale for some constant $n$. Fig. A1a shows the probability mass function of passive tracer $s$ for $n=1 / 6$, whereas Fig. A1b is for $n=1 / 30$. Small $\tau$ means that the passive tracer is relaxed to the reference $s\left(t=t_{0}\right)$ at a faster rate. The green lines in Fig. A1b demarcate the distance from respective levels computed as the updraft or subsidence conditionally averaged vertical velocity multiply by $\tau$. Thus, the green lines in Fig. A1 approximate the envelope of the average distance traversed by the passive tracer within the time window $\tau$. Although the envelopes of the average distance of updrafts and subsidence differ and therefore for the peak $s^{\prime}$ differ, the fractional contribution of updrafts and subsidence to total fluxes give similar results (Figs. A2a,c).

The instantaneous $s_{\text {peak }}^{\prime}$ for high- and low- $B_{0}$ runs of DNS are shown in Figs. A3a and A3b after $f(s ; z)$ has become quasi stationary. The variability of $s_{\text {peak }}^{\prime}, V a_{s}$, is defined as the ratio between the standard deviation of $s_{\text {peak }}^{\prime}(t)$ and the average of $s_{\text {peak }}^{\prime}(t)$. For the high- $B_{0}$ run, $V a_{s}$ is $0.047,0.13$, 0.026 , and 0.10 for $u^{\prime} w^{\prime u}, u^{\prime} w^{\prime d}, \theta^{\prime} w^{\prime u}$, and $\theta^{\prime} w^{\prime d}$, respectively. For the low- $B_{0}$ run, $V a_{s}$ is $0.074,0.15,0.073$, and 0.14 for $u^{\prime} w^{\prime u}, u^{\prime} w^{\prime d}, \theta^{\prime} w^{\prime u}$, and $\theta^{\prime} w^{\prime d}$, respectively.

\section{APPENDIX B}

\section{Sensitivity Test for LES}

\section{a. Grid sensitivity test of LES}

We tested LES with different resolutions $N_{x}=N_{y}=$ $N_{z}$ specified in the legend in Fig. B1b. The kinematic heat flux is $0.05 \mathrm{~K} \mathrm{~m} \mathrm{~s}^{-1}$, and the geostrophic wind speed 
(a)

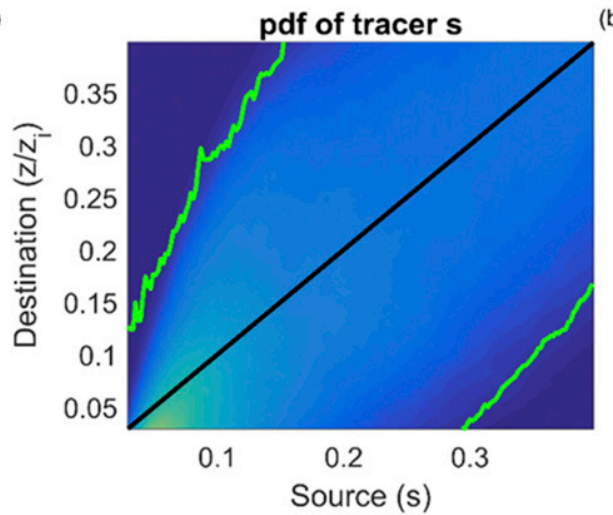

(b)

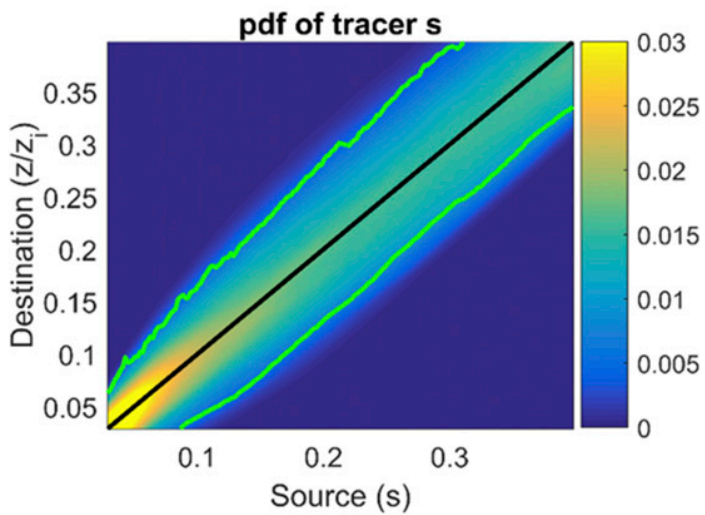

FIG. A1. The probability mass function of passive tracer $s$ for different relaxation rate: (a) $n=1 / 3$ and (b) $n=1 / 30$ for the DNS run low-buoyancy case.

is $8 \mathrm{~m} \mathrm{~s}^{-1}$. The averaging time for the data is approximately $800 \mathrm{~s}$ for a large-eddy turnover time defined as $z_{i} / w_{*}$. Note that $\phi_{m}$ and $\phi_{h}$ converge with resolution $512^{3}$, and therefore, we choose this grid structure for subsequent simulations. The oscillations (Fig. B2), which are characteristic of the overshoot discussed in the main text, are also reduced by increasing grid resolution. The values of $\phi_{m}$ for different simulations converge for small $-\zeta$; however, this may be due to the influence of the MOST-based closure imposed on the first level. Such convergence is not observed for $\phi_{h}$ since no MOSTbased wall model is imposed for the temperature.

\section{b. The pdf analysis of LES results}

The $\left\langle u^{\prime} w^{\prime} \mid s ; z\right\rangle_{N}$ and $\left\langle\theta^{\prime} w^{\prime} \mid s ; z\right\rangle_{N}$ for LES are shown in Fig. B3 for the low- $B_{0}$ case. The position of the peaks for updrafts scale with $z / z_{i}$, similar to DNS.

LES for the high- $B_{0}$ case also demonstrates similar features of $u^{\prime}$ of both signs present in the cores of regions of subsidence in Fig. B4. This shows that the mechanism (a)

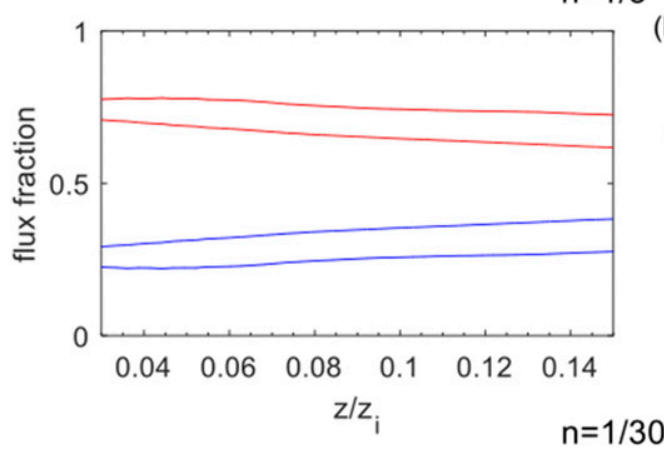

(c)

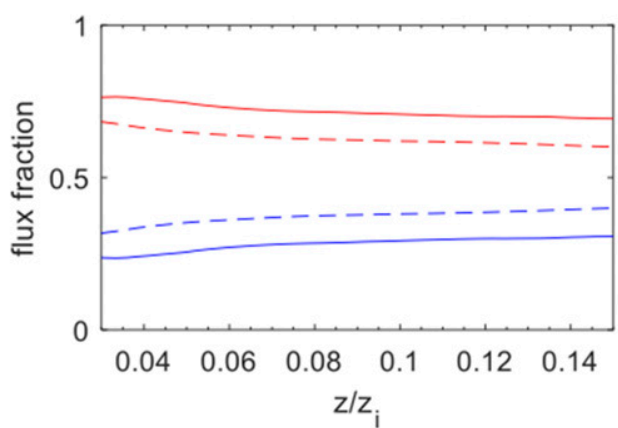

(b)

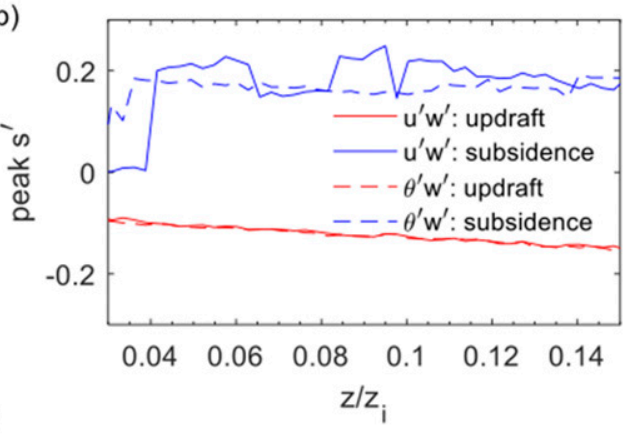

(d)

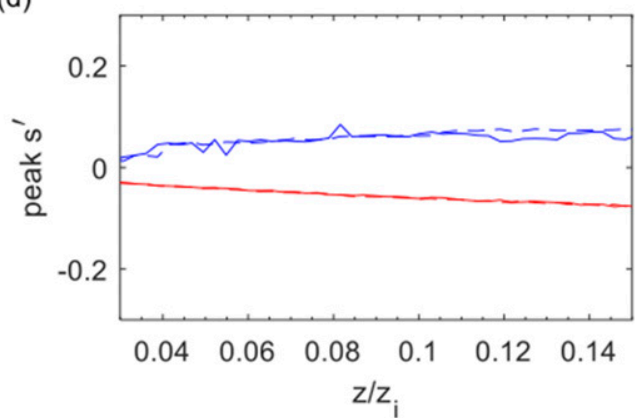

FIG. A2. Fraction of normalized fluxes for (a) $n=1 / 3$ and (c) $n=1 / 30$. Peak $s^{\prime}$ where the maximum of $X^{\prime} w_{N}^{\prime}$ occurs for $s^{\prime}<0$ (updrafts) or $s^{\prime}>0$ (downdrafts); (b) $n=1 / 3$ and (d) $b=1 / 30$. 

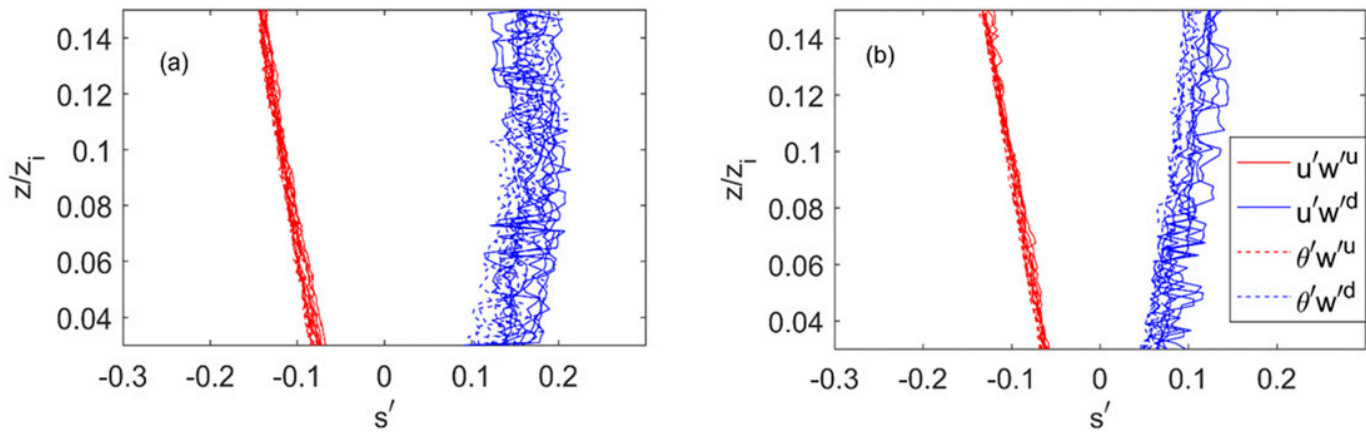

FIG. A3. Instantaneous $s_{\text {peak }}^{\prime}$ for (a) high-buoyancy DNS run and (b) low-buoyancy DNS run.

that induces inefficiency in momentum transfer by DNS, albeit at a low-shear Reynolds number for a highly convective case, is not biased by the Reynolds number effect.

\section{APPENDIX C}

\section{Nondimensional Analysis of DNS and LES}

The Navier-Stokes equations in the Boussinesq approximation are given as

$$
\begin{aligned}
\frac{\partial u_{i}}{\partial t}+u_{j} \frac{\partial u_{i}}{\partial x_{j}}= & -\frac{\partial p}{\partial x_{i}}+b \delta_{i 3}+f_{c}\left(u_{1}-G_{2}\right) \delta_{i 1} \\
& -f_{c}\left(u_{1}-G_{1}\right) \delta_{i 2}+\frac{\partial \tau_{i j}}{\partial x_{j}}, \\
\frac{\partial u_{j}}{\partial x_{j}}= & 0,
\end{aligned}
$$

$$
\frac{\partial b}{\partial t}+u_{j} \frac{\partial b}{\partial x_{j}}=-\frac{\partial q_{j}}{\partial x_{j}}
$$

where $u_{i}$ is the velocity component in the direction $I ; p$ is a modified pressure divided by the constant reference density; $b$ is the buoyancy defined as $g\left(\theta-\theta_{\text {ref }}\right) / \theta_{\text {ref }}$, where $\theta_{\text {ref }}$ is the reference value obtained by extrapolating the linear stratification of $\theta$ in the free atmosphere downward to the surface; $\tau$ is the viscous stress; and $q$ is the buoyancy flux. An alternative interpretation for $\tau$ and $q$ in the case of LES will be given as follows.

For DNS, the stress and buoyancy flux are given by

$$
\begin{aligned}
\tau_{i j}^{\mathrm{DNS}} & =\nu\left(\frac{\partial u_{i}}{\partial x_{j}}+\frac{\partial u_{j}}{\partial x_{i}}\right), \\
q_{j}^{\mathrm{DNS}} & =-\nu \operatorname{Pr}^{-1} \frac{\partial b}{\partial x_{j}} .
\end{aligned}
$$

Equations $(\mathrm{C} 1)-(\mathrm{C} 3)$ can be rewritten as

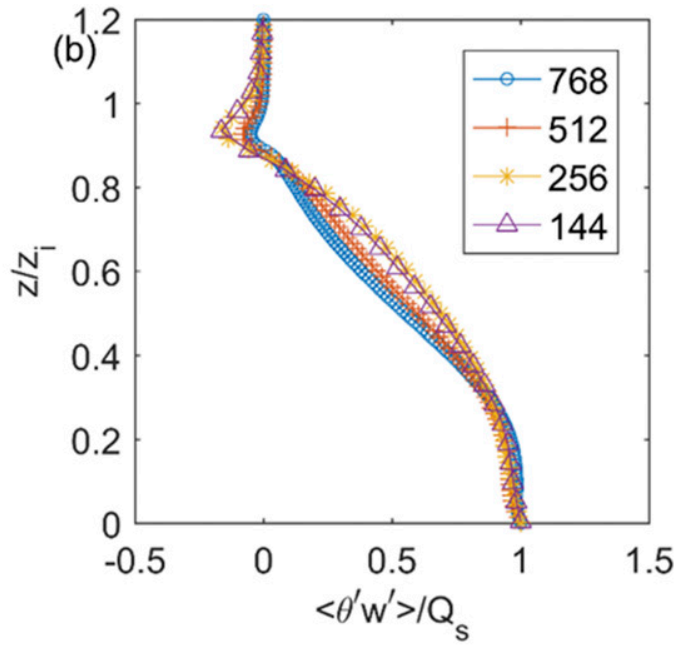

FIG. B1. Grid sensitivity test for (a) $\langle u\rangle / U_{g}$ and (b) $\left\langle\theta^{\prime} w^{\prime}\right\rangle$. 

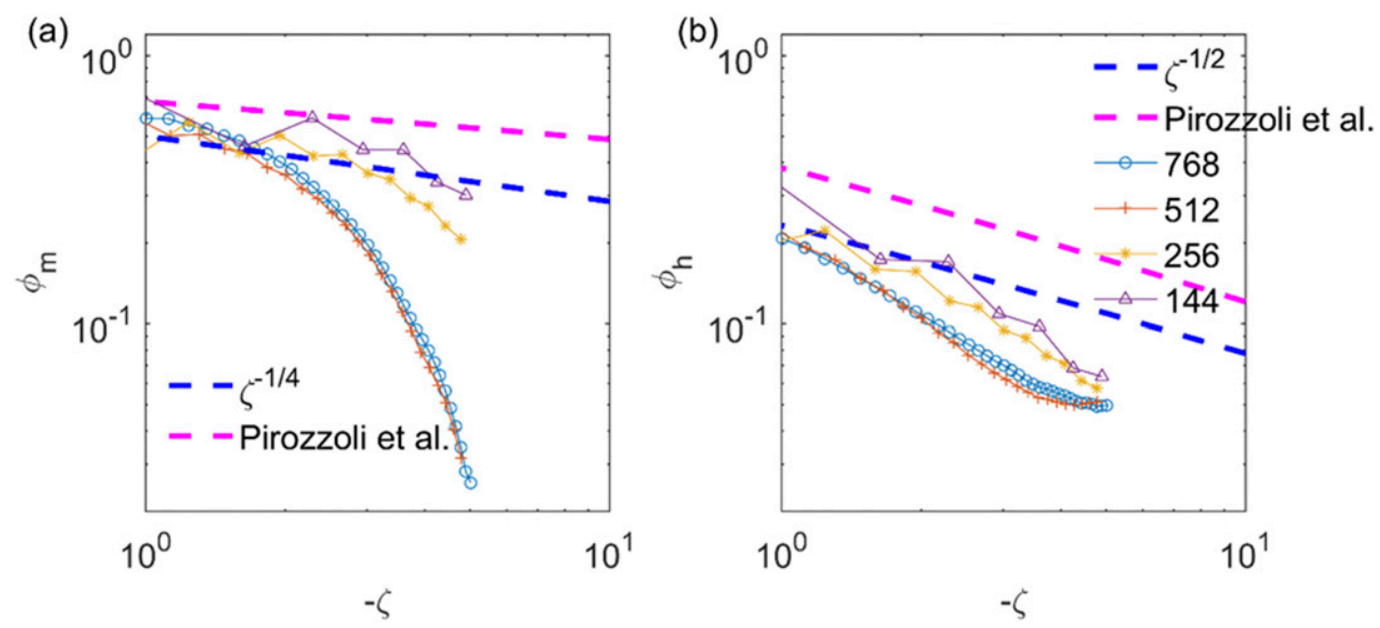

FIG. B2. Grid sensitivity test for (a) $\phi_{m}$ and (b) $\phi_{h}$.

$$
\begin{aligned}
\frac{\partial u_{i}}{\partial t}+u_{j} \frac{\partial u_{i}}{\partial x_{j}}= & -\frac{\partial p}{\partial x_{i}}+b \delta_{i 3}+f_{c}\left(u_{2}-G_{2}\right) \delta_{i 1} \\
& -f_{c}\left(u_{1}-G_{1}\right) \delta_{i 2}+\frac{\partial \tau_{i j}^{\mathrm{LES}}}{\partial x_{j}} \\
\frac{\partial u_{j}}{\partial x_{j}}= & 0 \\
\frac{\partial b}{\partial t}+u_{j} \frac{\partial b}{\partial x_{j}}= & -\frac{\partial q_{j}^{\mathrm{LES}}}{\partial x_{j}}
\end{aligned}
$$

where subgrid-scale closure schemes are needed for $\tau_{i j}^{\mathrm{LES}}$ and $q_{j}^{\mathrm{LES}}$ and it is understood that all variables are filtered variables and the tilde that represents the filtered variables is omitted for brevity.

(a)

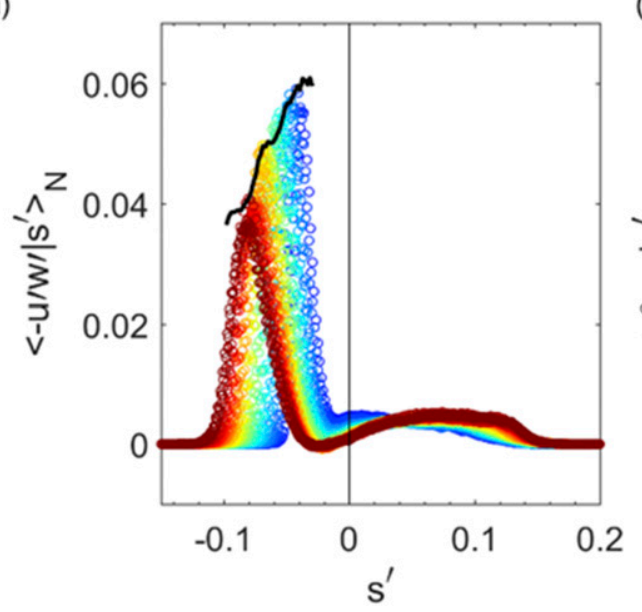

For LES, we can choose a general form of the subgrid-scale closure, and the tilde is added to emphasize that the variables are resolved ones in LES:

$$
\begin{aligned}
\tau_{i j}^{\mathrm{LES}} & =\nu_{\mathrm{SGS}}\left(\frac{\partial \tilde{u}_{i}}{\partial x_{j}}+\frac{\partial \tilde{u}_{j}}{\partial x_{i}}\right), \\
q_{j}^{\mathrm{LES}} & =-\nu_{\mathrm{SGS}} \operatorname{Pr}_{\mathrm{SGS}}^{-1} \frac{\partial \tilde{b}}{\partial x_{j}},
\end{aligned}
$$

where $\nu_{\mathrm{SGS}}$ is the subgrid-scale viscosity, which is given by

$$
\nu_{\mathrm{SGS}}=\left(C_{s} \Delta f\right)^{2}|\tilde{S}|,
$$

(b)

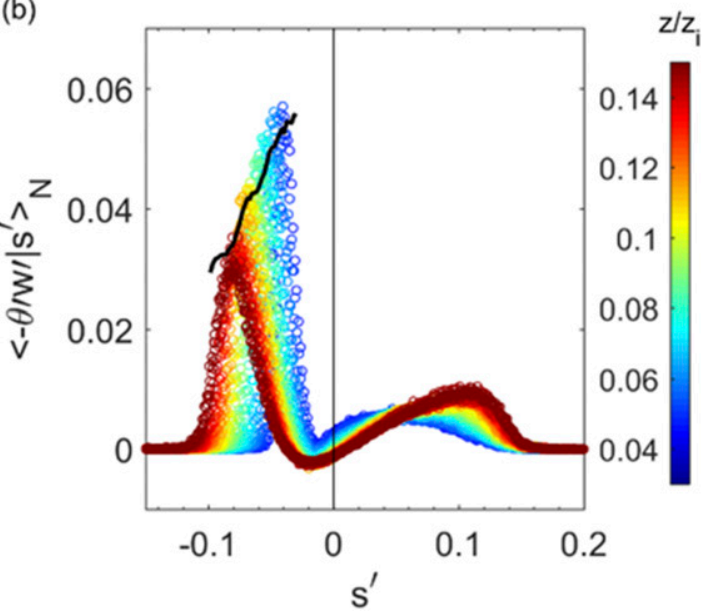

FIG. B3. The normalized flux contributions for the low-buoyancy LES run (a) $\left\langle u^{\prime} w^{\prime} \mid s^{\prime} ; z\right\rangle_{N}$ and (b) $\left\langle\theta^{\prime} w^{\prime} \mid s^{\prime} ; z\right\rangle_{N}$. The black line indicates the peak position given by the estimation $\kappa z / z_{i}$. 
(a)

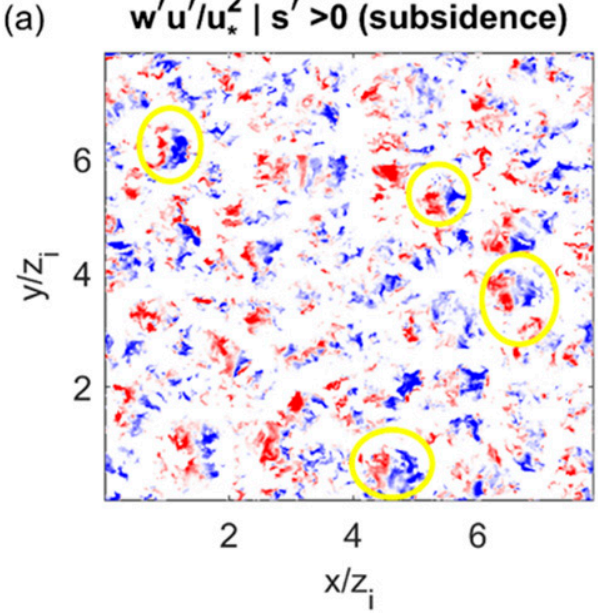

(b) $\quad \mathbf{w}^{\prime} \mathbf{u}^{\prime} / \mathbf{u}_{*}^{2} \mid \mathbf{s}^{\prime}<0$ (updraft)

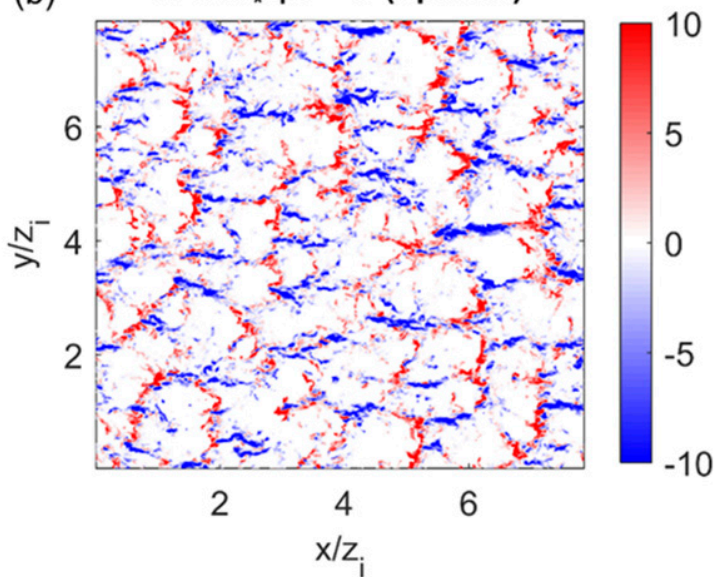

FIG. B4. The high-buoyancy LES run: $x-y$ cross section of $u^{\prime} w^{\prime}$ at $z / z_{i}=0.06$ conditioned on $s^{\prime}$; (a) $s^{\prime}>0$ represents subsidence and (b) $s^{\prime}<0$ represents an updraft.

where $C_{s}$ is the Smagorinsky coefficient, which can be evaluated based on different subgrid-scale models applied; $|\tilde{S}|$ is the magnitude of the filtered strain rate tensor; $\Delta f$ is the filter width in LES; and $\mathrm{Pr}^{\mathrm{SGS}}$ is the subgrid-scale Prandtl number.

The structure of the equations solved in DNS and LES is the same. Furthermore, we can use the same largescale characteristic velocity $U_{0}$ and length-scale $L_{c}$ for nondimensionalizing Eqs. $(\mathrm{C} 1)-(\mathrm{C} 3)$. The variables are made dimensionless using $U_{0}$ and $L_{c}$. Equations (C4)-(C8) in their dimensionless forms, where the hat denotes the nondimensionalized variable, are given by

$$
\begin{aligned}
\hat{\tau}_{i j}^{\mathrm{DNS}} & =\operatorname{Re}^{-1}\left(\frac{\partial \hat{u}_{i}}{\partial \hat{x}_{j}}+\frac{\partial \hat{u}_{j}}{\partial \hat{x}_{i}}\right), \\
\hat{q}_{j}^{\mathrm{DNS}} & =-(\operatorname{RePr})^{-1} \frac{\partial \hat{b}}{\partial \hat{x}_{j}},
\end{aligned}
$$

for DNS and for LES,

$$
\begin{aligned}
& \hat{\tau}_{i j}^{\mathrm{LES}}=\hat{\nu}_{\mathrm{SGS}}\left(\frac{\partial \hat{u}_{i}}{\partial \hat{x}_{j}}+\frac{\partial \hat{u}_{j}}{\partial \hat{x}_{i}}\right), \\
& \hat{q}_{j}^{\mathrm{LES}}=\hat{\nu}_{\mathrm{SGS}} \operatorname{Pr}_{\mathrm{SGS}}^{-1} \frac{\partial \hat{b}}{\partial x_{j}} .
\end{aligned}
$$

Specifically, nondimensionalizing $\Delta f$ in Eq. (C8) by $L_{c}$ leads to

$$
\hat{\nu}_{\mathrm{SGS}}=\left(\frac{C_{s} \Delta f}{L_{c}}\right)^{2}|\widetilde{\hat{S}}|=L r^{-2}|\widetilde{\hat{S}}|
$$

The nondimensional parameters are

$$
\operatorname{Re}=\frac{U_{0} L_{c}}{\nu}, \quad \operatorname{Pr}=\nu / \alpha, \quad L r=L_{c} /\left(C_{s} \Delta f\right),
$$

where $L_{r}$ can be considered as the length-scale separation in LES. Without loss of generality, $\operatorname{Pr}_{\mathrm{SGS}}$ is assumed to be a constant. For the buoyancy-driven CBL, we can define the velocity $U_{0}=\left(B_{0} L_{c}\right)^{1 / 3}$, which gives a reference dissipation rate $\epsilon_{0}=C_{\epsilon} U_{0}^{3} / L_{C}=C_{\epsilon} B_{0}$. The corresponding reference Kolmogorov scale is given as $\eta_{0}=\left(\nu^{3} / \varepsilon_{0}\right)^{1 / 4}=$ $\nu^{3 / 4} /\left(C \varepsilon B_{0}\right)^{1 / 4} \sim z_{\kappa}$. The Reynolds number is

$$
\operatorname{Re}=\left[L_{c} /\left(C_{\epsilon}^{1 / 4} \eta_{0}\right)\right]^{4 / 3}=\left(L_{c} / z_{\text {kappa }}\right)^{4 / 3} .
$$

Since the filter size $\Delta f$ in the LES is commensurate with the grid size, and the grid size in a DNS needs to be commensurate with the Kolmogorov-scale $\eta_{0}$ for the DNS to be resolved (no numerical artifacts or stable simulation), the "equivalent" DNS can be obtained from a given LES by

$$
\operatorname{Re}=\left(\gamma L_{r}\right)^{4 / 3}=\left[\gamma L_{c} /\left(C_{s} \Delta f\right)\right]^{4 / 3},
$$

where $\gamma=\left(C_{s} \Delta f\right) / z_{\kappa}$ is some constant of order 1 . For example, to estimate $\mathrm{Re}$ in an equivalent based on a given LES, we can take $L_{c}$ as the CBL depth with 400 points in the vertical direction (assume the grid is uniform) and $L_{c} / \Delta f \sim 200$ (if two points per filter size). Taking $C_{s} \sim 0.2$, this gives $\operatorname{Re} \sim 10^{4}$. The value of $\gamma$ cannot be obtained a priori; however, we could match the buoyancy Reynolds number $\mathrm{Re}_{o}$ with key steps shown as follows in order to determine $\gamma$ or $\beta$, where $z_{\kappa}=\beta \Delta f$ :

1) We run one LES with a given geostrophic wind and surface heat flux for a given resolution (e.g., $512^{3}$ ). 
2) Assuming $\beta=1$, one can compute $\operatorname{Re}_{o}^{\mathrm{LES}}$, and since $\mathrm{Re}_{o}^{\mathrm{LES}}=\mathrm{Re}_{o}^{\mathrm{DNS}}\left[\right.$ i.e., $\left(B_{0} / N^{3}\right)_{\mathrm{LES}}^{1 / 2} / z_{\kappa_{\mathrm{eff}}}=\left(B_{0} / N^{3}\right)_{\mathrm{LES}}^{1 / 2} /$ $\left.\beta \Delta_{f}=\operatorname{Re}_{o}^{\mathrm{LES}}=\operatorname{Re}_{o}^{\mathrm{DNS}}=\left(B_{0} / N^{3}\right)_{\mathrm{DNS}}^{1 / 2} / z_{\kappa}, \beta=1\right]$, we run DNS at this buoyancy Reynolds number. The DNS run should be resolved as indicated by the ratio between the Kolmogorov-scale $\eta_{k}$ and the grid size $\Delta$ to be 1.0-1.5 (Moeng and Wyngaard 1984, 1989; Wyngaard and Brost 1984; Wyngaard and Weil 1991; Wyngaard and Moeng 1992).

3) If $\eta_{k} \ll 1.5 \Delta z$, DNS is underresolved, which means that $\mathrm{Re}_{o}^{\mathrm{LES} / \mathrm{DNS}}$ is too large and $\beta$ needs to be increased; vice versa if $\eta_{k} \ll 1.5 \Delta z$.

4) Repeat 2 and 3 until the $\eta_{k} \sim 1.0-1.5 \Delta$ near the surface.

After some trial and error, we found that $\beta=1.05$ was the appropriate parameter, such that DNS is not underresolved or overresolved, which gives $\mathrm{Re}_{o}=15$ as the equivalent buoyancy Reynolds number in both LES and DNS.

\section{REFERENCES}

Adrian, R. J., 2007: Hairpin vortex organization in wall turbulence. Phys. Fluids, 19, 041301, https://doi.org/10.1063/1.2717527.

Albertson, J. D., 1996: Large eddy simulation of land-atmosphere interaction. Ph.D. dissertation, University of California, Davis, 185 pp.

_ stress: Implications for land-atmosphere interaction over complex terrain. Water Resour. Res., 35, 2121-2132, https:// doi.org/10.1029/1999WR900094.

Andreas, E. L, and B. B. Hicks, 2002: Comments on "Critical test of the validity of Monin-Obukhov similarity during convective conditions." J. Atmos. Sci., 59, 2605-2607, https://doi.org/ 10.1175/1520-0469(2002)059<2605:COCTOT>2.0.CO;2.

Antonia, R. A., and A. J. Chambers, 1978: Note on the temperature ramp structure in the marine surface layer. Bound.-Layer Meteor., 15, 347-355, https://doi.org/10.1007/BF02652606.

, C. A. Friehe, and C. W. Van Atta, 1979: Temperature ramps in the atmospheric surface layer. J. Atmos. Sci., 36, 99-108, https://doi.org/10.1175/1520-0469(1979)036<0099: TRITAS $>2.0 . \mathrm{CO} ; 2$.

Baars, W. J., N. Hutchins, and I. Marusic, 2017: Reynolds number trend of hierarchies and scale interactions in turbulent boundary layers. Philos. Trans. Roy. Soc. London, 375A, 20160077, https://doi.org/10.1098/rsta.2016.0077.

Banerjee, T., D. Li, J.-Y. Juang, and G. Katul, 2016: A spectral budget model for the longitudinal turbulent velocity in the stable atmospheric surface layer. J. Atmos. Sci., 73, 145-166, https://doi.org/10.1175/JAS-D-15-0066.1.

Beljaars, A. C. M., and A. A. M. Holtslag, 1991: Flux parameterization over land surfaces for atmospheric models. J. Appl. Meteor., 30, 327-341, https://doi.org/10.1175/1520-0450(1991)030<0327: FPOLSF $>2.0 . \mathrm{CO} ; 2$.

Berg, L. K., and R. B. Stull, 2004: Parameterization of joint frequency distributions of potential temperature and water vapor mixing ratio in the daytime convective boundary layer. J. Atmos. Sci., 61, 813-828, https://doi.org/10.1175/ 1520-0469(2004)061<0813:POJFDO>2.0.CO;2.
Berkooz, G., P. Holmes, and J. L. Lumley, 1993: The proper orthogonal decomposition in the analysis of turbulent flows. Annu. Rev. Fluid Mech., 25, 539-575, https://doi.org/10.1146/ annurev.fl.25.010193.002543.

Betts, A. K., and C. Jakob, 2002: Evaluation of the diurnal cycle of precipitation, surface thermodynamics, and surface fluxes in the ECMWF model using LBA data. J. Geophys. Res., 107, 8045, https://doi.org/10.1029/2001JD000427.

Betts, R. A., 2000: Offset of the potential carbon sink from boreal forestation by decreases in surface albedo. Nature, 408, 187190, https://doi.org/10.1038/35041545.

Bose, S. T., P. Moin, and D. You, 2010: Grid-independent largeeddy simulation using explicit filtering. Phys. Fluids, 22, 105103, https://doi.org/10.1063/1.3485774.

Bou-Zeid, E., C. Meneveau, and M. Parlange, 2005: A scaledependent Lagrangian dynamic model for large eddy simulation of complex turbulent flows. Phys. Fluids, 17, 025105, https://doi.org/10.1063/1.1839152.

Brasseur, J. G., and T. Wei, 2010: Designing large-eddy simulation of the turbulent boundary layer to capture law-of-the-wall scaling. Phys. Fluids, 22, 021303, https://doi.org/10.1063/1.3319073.

Brunet, Y., and M. R. Irvine, 2000: The control of coherent eddies in vegetation canopies: Streamwise structure spacing, canopy shear scale and atmospheric stability. Bound.-Layer Meteor., 94, 139-163, https://doi.org/10.1023/A:1002406616227.

Businger, J. A., 1973: A note on free convection. Bound.-Layer Meteor., 4, 323-326, https://doi.org/10.1007/BF02265241.

, and S. P. Oncley, 1990: Flux measurement with conditional sampling. J. Atmos. Oceanic Technol., 7, 349-352, https://doi.org/ 10.1175/1520-0426(1990)007<0349:FMWCS >2.0.CO;2.

_ J. C. Wyngaard, Y. Izumi, and E. F. Bradley, 1971: Fluxprofile relationships in the atmospheric surface layer. J. Atmos. Sci., 28, 181-189, https://doi.org/10.1175/1520-0469 (1971)028<0181:FPRITA $>2.0 . C O ; 2$.

Calaf, M., M. Hultmark, H. J. Oldroyd, V. Simeonov, and M. B. Parlange, 2013: Coherent structures and the $k^{-1}$ spectral behaviour. Phys. Fluids, 25, 125107, https://doi.org/10.1063/1.4834436.

Cancelli, D. M., M. Chamecki, and N. L. Dias, 2014: A large-eddy simulation study of scalar dissimilarity in the convective atmospheric boundary layer. J. Atmos. Sci., 71, 3-15, https://doi.org/ 10.1175/JAS-D-13-0113.1.

Chung, D., and G. Matheou, 2012: Direct numerical simulation of stationary homogeneous stratified sheared turbulence. J. Fluid Mech., 696, 434-467, https://doi.org/10.1017/jfm.2012.59.

Coleman, G. N., J. H. Ferziger, and P. R. Spalart, 1994: A numerical study of the convective boundary layer. Bound.-Layer Meteor., 70, 247-272, https://doi.org/10.1007/BF00709121.

Couvreux, F., F. Hourdin, and C. Rio, 2010: Resolved versus parametrized boundary-layer plumes. Part I: A parametrizationoriented conditional sampling in large-eddy simulations. Bound.-Layer Meteor., 134, 441-458, https://doi.org/10.1007/ s10546-009-9456-5.

Delire, C., J. A. Foley, and S. Thompson, 2004: Long-tern variability in a coupled atmosphere-biosphere model. J. Climate, 17, 3947-3959, https://doi.org/10.1175/1520-0442(2004)017<3947: LVIACA $>2.0 . \mathrm{CO} ; 2$.

Dyer, A. J., 1974: A review of flux-profile relationships. Bound.Layer Meteor., 7, 363-372, https://doi.org/10.1007/BF00240838.

Farge, M., G. Pellegrino, and K. Schneider, 2001: Coherent vortex extraction in 3D turbulent flows using orthogonal wavelets. Phys. Rev. Lett., 87, 054501, https://doi.org/10.1103/PhysRevLett.87.054501.

Fedorovich, E., and A. Shapiro, 2009: Turbulent natural convection along a vertical plate immersed in a stably 
stratified fluid. J. Fluid Mech., 636, 41-57, https://doi.org/ 10.1017/S0022112009007757.

Findell, K. L., P. Gentine, B. R. Lintner, and C. Kerr, 2011: Probability of afternoon precipitation in eastern United States and Mexico enhanced by high evaporation. Nat. Geosci., 4 , 434-439, https://doi.org/10.1038/NGEO1174.

Foken, T., 2006: 50 years of the Monin-Obukhov similarity theory. Bound.-Layer Meteor., 119, 431-447, https://doi.org/10.1007/ s10546-006-9048-6.

Gao, Z., H. Liu, E. S. Russell, J. Huang, T. Foken, and S. P. Oncley, 2016: Large eddies modulating flux convergence and divergence in a disturbed unstable atmospheric surface layer. J. Geophys. Res. Atmos., 121, 1475-1492, https://doi.org/ 10.1002/2015JD024529.

Garcia, J. R., and J. P. Mellado, 2014: The two-layer structure of the entrainment zone in the convective boundary layer. J. Atmos. Sci., 71, 1935-1955, https://doi.org/10.1175/JAS-D-13-0148.1.

Gentine, P., D. Entekhabi, A. Chehbouni, G. Boulet, and B. Duchemin, 2007: Analysis of evaporative fraction diurnal behaviour. Agric. For. Meteor., 143, 13-29, https://doi.org/ 10.1016/j.agrformet.2006.11.002.

— - — , and J. Polcher, 2011a: The diurnal behavior of evaporative fraction in the soil-vegetation-atmospheric boundary layer continuum. J. Hydrometeor., 12, 1530-1546, https://doi.org/ 10.1175/2011JHM1261.1.

— J. Jolcher, and D. Entekhabi, 2011b: Harmonic propagation of variability in surface energy balance within a coupled soilvegetation-atmosphere system. Water Resour. Res., 47, W05525, https://doi.org/10.1029/2010WR009268.

_ A. A. M. Holtslag, F. D'Andrea, and M. Ek, 2013: Surface and atmospheric controls on the onset of moist convection over land. J. Hydrometeor., 14, 1443-1462, https://doi.org/ 10.1175/JHM-D-12-0137.1.

Ghannam, K., T. Duman, S. T. Salesky, M. Chamecki, and G. Katul, 2017: The non-local character of turbulence asymmetry in the convective atmospheric boundary layer. Quart. J. Roy. Meteor. Soc., 143, 494-507, https://doi.org/10.1002/qj.2937.

Green, M. A., C. W. Rowley, and G. Haller, 2007: Detection of Lagrangian coherent structures in three-dimensional turbulence. J. Fluid Mech., 572, 111-120, https://doi.org/10.1017/ S0022112006003648.

Haller, G., 2015: Lagrangian coherent structures. Annu. Rev. Fluid Mech., 47, 137-162, https://doi.org/10.1146/annurevfluid-010313-141322.

—_, and G. Yuan, 2000: Lagrangian coherent structures and mixing in two-dimensional turbulence. Physica D, 147, 352370, https://doi.org/10.1016/S0167-2789(00)00142-1.

Head, M. R., and P. Bandyopadhyay, 1981: New aspects of turbulent boundary-layer structure. J. Fluid Mech., 107, 297-338, https://doi.org/10.1017/S0022112081001791.

Högström, U., 1988: Non-dimensional wind and temperature profiles in the atmospheric surface layer: A re-evaluation. Bound.-Layer Meteor., 42, 55-78, https://doi.org/10.1007/BF00119875.

Hommema, S. E., and R. J. Adrian, 2003: Packet structure of surface eddies in the atmospheric boundary layer. Bound.-Layer Meteor., 106, 147-170, https://doi.org/10.1023/A:1020868132429.

Huang, J., M. Cassiani, and J. D. Albertson, 2009: Analysis of coherent structures within the atmospheric boundary layer. Bound.-Layer Meteor., 131, 147-171, https://doi.org/10.1007/ s10546-009-9357-7.

Hunt, J. C. R., and J. F. Morrison, 2000: Eddy structure in turbulent boundary layers. Eur. J. Mech., 19B, 673-694, https://doi.org/ 10.1016/S0997-7546(00)00129-1.
Johansson, C., A.-S. Smedman, U. Högström, J. G. Brasseur, and S. Khanna, 2001: Critical test of the validity of Monin-Obukhov similarity during convective conditions. J. Atmos. Sci., 58, 1549-1566, https://doi.org/10.1175/1520-0469(2001)058<1549: CTOTVO $>2.0 . \mathrm{CO} ; 2$.

Jonker, H. J. J., P. G. Duynkerke, and J. W. M. Cuijpers, 1999: Mesoscale fluctuations in scalars generated by boundary layer convection. J. Atmos. Sci., 56, 801-808, https://doi.org/10.1175/ 1520-0469(1999)056<0801:MFISGB >2.0.CO;2.

Kader, B. A., and A. M. Yaglom, 1990: Mean fields and fluctuation moments in unstably stratified turbulent boundary layers. J. Fluid Mech., 212, 637-662, https://doi.org/10.1017/ S0022112090002129.

Kaimal, J. C., and J. J. Finnigan, 1994: Atmospheric Boundary Layer Flows: Their Structure and Measurement. Oxford University Press, 289 pp.

Karrasch, D., and G. Haller, 2013: Do finite-size Lyapunov exponents detect coherent structures? Chaos, 23, 043126, https:// doi.org/10.1063/1.4837075.

Katul, G. G., 1994: A model for sensible heat flux probability density function for near-neutral and slightly-stable atmospheric flows. Bound.-Layer Meteor., 71, 1-20, https://doi.org/ 10.1007/BF00709217.

, J. D. Albertson, M. B. Parlange, C.-I. Hsieh, P. S. Conklin, J. T. Sigmon, and K. R. Knoerr, 1996: The "inactive" eddy motion and the large-scale turbulent pressure fluctuations in the dynamic sublayer. J. Atmos. Sci., 53, 2512-2524, https://doi.org/ 10.1175/1520-0469(1996)053<2512:TEMATL >2.0.CO;2.

— A. Porporato, E. Daly, A. C. Oishi, H. S. Kim, P. C. Stoy, J. Y. Juang, and M. B. Siqueira, 2007: On the spectrum of soil moisture from hourly to interannual scales. Water Resour. Res., 43, W05428, https://doi.org/10.1029/2006WR005356.

_ A. G. Konings, and A. Porporato, 2011: Mean velocity profile in a sheared and thermally stratified atmospheric boundary layer. Phys. Rev. Lett., 107, 268502, https://doi.org/10.1103/ PhysRevLett.107.268502.

, D. Li, M. Chameki, and E. Bou-Zeid, 2013: Mean scalar concentration profile in a sheared and thermally stratified atmospheric surface layer. Phys. Rev., 87E, 023004, https://doi.org/ 10.1103/PhysRevE.87.023004.

—, A. Porporato, S. Shah, and E. Bou-Zeid, 2014: Two phenomenological constants explain similarity laws in stably stratified turbulence. Phys. Rev., 89E, 023007, https://doi.org/ 10.1103/PhysRevE.89.023007.

Khanna, S., and J. G. Brasseur, 1997: Analysis of Monin-Obukhov similarity from large-eddy simulation. J. Fluid Mech., 345, 251-286, https://doi.org/10.1017/S0022112097006277.

— , and — 1998: Three-dimensional buoyancy- and shear-induced local structure of the atmospheric boundary layer. J. Atmos. Sci., 55, 710-743, https://doi.org/10.1175/1520-0469(1998)055<0710: TDBASI $>2.0 . \mathrm{CO} ; 2$.

Kumar, V., J. Kleissl, C. Meneveau, and M. B. Parlange, 2006: Large-eddy simulation of a diurnal cycle of the atmospheric boundary layer: Atmospheric stability and scaling issues. Water Resour. Res., 42, W06D09, https://doi.org/10.1029/ 2005WR004651.

Laubach, J., and K. G. McNaughton, 2009: Scaling properties of temperature spectra and heat-flux cospectra in the surface friction layer beneath an unstable outer layer. Bound.-Layer Meteor., 133, 219-252, https://doi.org/10.1007/ s10546-009-9422-2. 
Lenschow, D. H., and P. L. Stephens, 1980: The role of thermals in the convective boundary layer. Bound.-Layer Meteor., 19, 509-532, https://doi.org/10.1007/BF00122351.

Li, D., and E. Bou-Zeid, 2011: Coherent structures and the dissimilarity of turbulent transport of momentum and scalars in the unstable atmospheric surface layer. Bound.-Layer Meteor., 140, 243-262, https://doi.org/10.1007/s10546-011-9613-5. , G. G. Katul, and E. Bou-Zeid, 2012: Mean velocity and temperature profiles in a sheared diabatic turbulent boundary layer. Phys. Fluids, 24, 105105, https://doi.org/10.1063/1.4757660.

Li, Q., E. Bou-Zeid, W. Anderson, S. Grimmond, and M. Hultmark, 2016: Quality and reliability of LES of convective scalar transfer at high Reynolds numbers. Int. J. Heat Mass Transfer, 102, 959970, https://doi.org/10.1016/j.ijheatmasstransfer.2016.06.093.

Mahrt, L., 1991: Eddy asymmetry in the sheared heated boundary layer. J. Atmos. Sci., 48, 472-492, https://doi.org/10.1175/15200469(1991)048<0472:EAITSH $>2.0$. CO;2.

Maronga, B., and J. Reuder, 2017: On the formulation and universality of Monin-Obukhov similarity functions for mean gradients and standard deviations in the unstable surface layer: Results from surface-layer-resolving large-eddy simulations. J. Atmos. Sci., 74, 989-1010, https://doi.org/10.1175/JAS-D-16-0186.1.

Marusic, I., R. Mathis, and N. Hutchins: 2010: Predictive model for wall-bounded turbulent flow. Science, 329, 193-196, https:// doi.org/10.1126/science.1188765.

Mathis, R., N. Hutchins, and I. Marusic, 2011: A predictive innerouter model for streamwise turbulence statistics in wallbounded flows. J. Fluid Mech., 681, 537-566, https://doi.org/ 10.1017/jfm.2011.216.

McColl, K. A., G. G. Katul, P. Gentine, and D. Entekhabi, 2016: Mean-velocity profile of smooth channel flow explained by a cospectral budget model with wall-blockage. Phys. Fluids, 28, 035107, https://doi.org/10.1063/1.4943599.

—, C. C. van Heerwaarden, G. G. Katul, P. Gentine, and D. Entekhabi, 2017: Role of large eddies in the breakdown of the Reynolds analogy in an idealized mildly unstable atmospheric surface layer. Quart. J. Roy. Meteor. Soc., 143, 21822197, https://doi.org/10.1002/qj.3077.

McNaughton, K. G., 2004: Attached eddies and production spectra in the atmospheric logarithmic layer. Bound.-Layer Meteor., 111, 1-18, https://doi.org/10.1023/B:BOUN.0000010997.51745.0f.

_ , and Y. Brunet, 2002: Townsend's hypothesis, coherent structures and Monin-Obukhov similarity. Bound.-Layer Meteor., 102, 161-175, https://doi.org/10.1023/A:1013171312407.

_, R. J. Clement, and J. B. Moncrieff, 2007: Scaling properties of velocity and temperature spectra above the surface friction layer in a convective atmospheric boundary layer. Nonlinear Processes Geophys., 14, 257-271, https://doi.org/10.5194/npg-14-257-2007.

McPhee, M. G., 1994: On the turbulent mixing length in the oceanic boundary layer. J. Phys. Oceanogr., 24, 2014-2031, https://doi.org/ 10.1175/1520-0485(1994)024<2014:OTTMLI > 2.0.CO;2.

Mellado, J. P., C. C. van Heerwaarden, and J. R. Garcia, 2016: Near-surface effects of free atmosphere stratification in free convection. Bound.-Layer Meteor., 159, 69-95, https://doi.org/ 10.1007/s10546-015-0105-x.

— C. S. Bretherton, B. Stevens, and M. C. Wyant, 2018: DNS and LES for simulating stratocumulus: Better together. J. Adv. Model. Earth Syst., 10, 1421-1438, https://doi.org/10.1029/2018MS001312.

Mestayer, P. G., C. H. Gibson, M. F. Coantic, and A. S. Patel, 1976: Local anisotropy in heated and cooled turbulent boundary layers. Phys. Fluids, 19, 1279-1287, https://doi.org/10.1063/1.861649.

Moeng, C.-H., 1984: A large-eddy-simulation model for the study of planetary boundary-layer turbulence. J. Atmos. Sci., 41,
2052-2062, https://doi.org/10.1175/1520-0469(1984)041<2052: ALESMF $>2.0 . \mathrm{CO} ; 2$.

_ and J. C. Wyngaard, 1984: Statistics of conservative scalars in the convective boundary layer. J. Atmos. Sci., 41, 3161-3169, https:// doi.org/10.1175/1520-0469(1984)041<3161:SOCSIT>2.0.CO;2.

— , and — 1988: Spectral analysis of large-eddy simulations of the convective boundary layer. J. Atmos. Sci., 45, 3573-3587, https:// doi.org/10.1175/1520-0469(1988)045<3573:SAOLES >2.0.CO;2.

— and _ 1989: Evaluation of turbulent transport and dissipation closures in second-order modeling. J. Atmos. Sci., 46, 2311-2330, https://doi.org/10.1175/1520-0469(1989)046<2311: EOTTAD $>2.0 . \mathrm{CO} ; 2$.

—, and P. P. Sullivan, 1994: A comparison of shear- and buoyancy-driven planetary boundary layer flows. J. Atmos. Sci., 51, 999-1022, https://doi.org/10.1175/1520-0469(1994) 051<0999:ACOSAB > 2.0.CO;2.

Monin, A. S., and A. M. Obukhov, 1954: Basic laws of turbulent mixing in the atmosphere near the ground. Tr. Geofiz. Inst., Akad. Nauk SSSR, 24, 163-187.

— chanics of Turbulence. Vol. 1. MIT Press, $782 \mathrm{pp}$.

Morrison, J. F., 2007: The interaction between inner and outer regions of turbulent wall-bounded flow. Philos. Trans. Roy. Soc., 365A, 683-698, https://doi.org/10.1098/rsta.2006.1947.

Nieuwstadt, F. T. M., P. J. Mason, C. H. Moeng, and U. Schumann, 1993: Large-eddy simulation of the convective boundary layer: A comparison of four computer codes. Turbulent Shear Flows 8 , Springer, 343-367.

Notaro, M., 2008: Statistical identification of global hot spots in soil moisture feedbacks among IPCC AR4 models. J. Geophys. Res., 113, D09101, https://doi.org/10.1029/2007JD009199.

Okamoto, N., K. Yoshimatsu, K. Schneider, M. Farge, and Y. Kaneda, 2007: Coherent vortices in high resolution direct numerical simulation of homogeneous isotropic turbulence: A wavelet viewpoint. Phys. Fluids, 19, 115109, https://doi.org/ 10.1063/1.2771661.

Orszag, S. A., 1971: On the elimination of aliasing in finite difference schemes by filtering high-wavenumber components. J. Atmos. Sci., 28, 1074, https://doi.org/10.1175/1520-0469(1971)028<1074: OTEOAI $>2.0 . \mathrm{CO} ; 2$.

Panofsky, H. A., and J. Dutton, 1984: Atmospheric Turbulence: Models and Methods for Engineering Applications. Wiley, 397 pp. -, H. Tennekes, D. H. Lenschow, and J. C. Wyngaard, 1977: The characteristics of turbulent velocity components in the surface layer under convective conditions. Bound.-Layer Meteor., 11, 355-361, https://doi.org/10.1007/BF02186086.

Park, S.-B., P. Gentine, K. Schneider, and M. Farge, 2016: Coherent structures in the boundary and cloud layers: Role of updrafts, subsiding shells, and environmental subsidence. J. Atmos. Sci., 73, 1789-1814, https://doi.org/10.1175/JAS-D-15-0240.1.

- T. Heus, and P. Gentine, 2017: Role of convective mixing and evaporative cooling in shallow convection. J. Geophys. Res. Atmos., 122, 5351-5363, https://doi.org/10.1002/2017JD026466.

- S. Böing, and P. Gentine, 2018: Role of surface friction on shallow nonprecipitating convection. J. Atmos. Sci., 75, 163 178, https://doi.org/10.1175/JAS-D-17-0106.1.

Patton, E. G., P. P. Sullivan, R. H. Shaw, J. J. Finnigan, and J. C. Weil, 2016: Atmospheric stability influences on coupled boundary layer and canopy turbulence. J. Atmos. Sci., 73, 1621-1647, https:// doi.org/10.1175/JAS-D-15-0068.1.

Perry, A. E., and M. S. Chong, 1982: On the mechanism of wall turbulence. J. Fluid Mech., 119, 173-217, https://doi.org/10.1017/ S0022112082001311. 
Pirozzoli, S., M. Bernardini, R. Verzicco, and P. Orlandi, 2017: Mixed convection in turbulent channels with unstable stratification. J. Fluid Mech., 821, 482-516, https://doi.org/10.1017/jfm.2017.216.

Provenzale, A., 1999: Transport by coherent barotropic vortices. Annu. Rev. Fluid Mech., 31, 55-93, https://doi.org/10.1146/ annurev.fluid.31.1.55.

Rockwood, M., Y. Huang, and M. Green, 2018: Tracking coherent structures in massively-separated and turbulent flows. Phys. Rev. Fluids, 3, 014702, https://doi.org/10.1103/PhysRevFluids.3.014702.

Rogallo, R. S., and P. Moin, 1984: Numerical simulation of turbulent flows. Annu. Rev. Fluid Mech., 16, 99-137, https://doi.org/ 10.1146/annurev.fl.16.010184.000531.

Salesky, S. T., and M. Chamecki, 2012: Random errors in turbulence measurements in the atmospheric surface layer: Implications for Monin-Obukhov similarity theory. J. Atmos. Sci., 69, 3700-3714, https://doi.org/10.1175/JAS-D-12-096.1.

,-- , and E. Bou-Zeid, 2017: On the nature of the transition between roll and cellular organization in the convective boundary layer. Bound.-Layer Meteor., 163, 41-68, https://doi.org/10.1007/ s10546-016-0220-3.

Schumann, U., and C.-H. Moeng, 1991a: Plume fluxes in clear and cloudy convective boundary layers. J. Atmos. Sci., 48 , 1746-1757, https://doi.org/10.1175/1520-0469(1991)048<1746: PFICAC $>2.0 . \mathrm{CO} ; 2$

$\longrightarrow$, and - 1991b: Plume budges in clear and cloudy convective boundary layers. J. Atmos. Sci., 48, 1758-1770, https://doi.org/ 10.1175/1520-0469(1991)048<1758:PBICAC > 2.0.CO;2.

Seneviratne, S. I., T. Corti, E. L. Davin, M. Hirschi, E. B. Jaeger, I. Lehner, B. Orlowsky, and A. J. Teuling, 2010: Investigating soil moisture-climate interactions in a changing climate: A review. Earth-Sci. Rev., 99, 125-161, https://doi.org/10.1016/ j.earscirev.2010.02.004.

Shah, S., and E. Bou-Zeid, 2014a: Very-large-scale motions in the atmospheric boundary layer educed by snapshot proper orthogonal decomposition. Bound.-Layer Meteor., 153, 355-387, https://doi.org/10.1007/s10546-014-9950-2.

— Ekman layers with increasing static stability: Modifications to the bulk structure and second-order statistics. J. Fluid Mech., 760, 494-539, https://doi.org/10.1017/jfm.2014.597.

Siebesma, A. P., P. M. M. Soares, and J. Teixeira, 2007: A combined eddy-diffusivity mass-flux approach for the convective boundary layer. J. Atmos. Sci., 64, 1230-1248, https://doi.org/10.1175/ JAS3888.1.

Stull, R. B., 1984: Transilient turbulence theory. Part I: The concept of eddy-mixing across finite distances. J. Atmos. Sci., 41, 3351-3367, https://doi.org/10.1175/1520-0469(1984)041<3351: TTTPIT $>2.0 . \mathrm{CO} ; 2$.

__ 1988: An Introduction to Boundary Layer Meteorology. Kluwer Academic, 666 pp.

_ 1993: Review of non-local mixing in turbulent atmospheres: Transilient turbulence theory. Bound.-Layer Meteor., 62, 2196, https://doi.org/10.1007/BF00705546.

Sullivan, P. P., and E. G. Patton, 2011: The effect of mesh resolution on convective boundary layer statistics and structures generated by large-eddy simulation. J. Atmos. Sci., 68, 23952415, https://doi.org/10.1175/JAS-D-10-05010.1.

Sun, J., D. H. Lenschow, M. A. LeMone, and L. Mahrt, 2016: The role of large-coherent-eddy transport in the atmospheric surface layer based on CASES-99 observations. Bound.-Layer Meteor., 160, 83-111, https://doi.org/10.1007/s10546-016-0134-0.
Thomas, C., and T. Foken, 2005: Detection of long-term coherent exchange over spruce forest using wavelet analysis. Theor. Appl. Climatol., 80, 91-104, https://doi.org/10.1007/s00704-004-0093-0.

Townsend, A. A., 1959: Temperature fluctuations over a heated horizontal surface. J. Fluid Mech., 5, 209-241, https://doi.org/ 10.1017/S0022112059000167.

_, 1961: Equilibrium layers and wall turbulence. J. Fluid. Mech., 11, 97-120, https://doi.org/10.1017/S0022112061000883.

1976: The Structure of Turbulent Shear Flow. Cambridge University Press, $444 \mathrm{pp}$

Wilczak, J. M., 1984: Large-scale eddies in the unstably stratified atmospheric surface layer. Part I: Velocity and temperature structure. J. Atmos. Sci., 41, 3537-3550, https://doi.org/ 10.1175/1520-0469(1984)041<3537:LSEITU>2.0.CO;2.

— , and J. E. Tillman, 1980: The three-dimensional structure of convection in the atmospheric surface layer. J. Atmos. Sci., 37, 2424-2443, https://doi.org/10.1175/1520-0469(1980)037<2424: TTDSOC $>2.0 . \mathrm{CO} ; 2$

Wilczek, M., B. Kadoch, K. Schneider, R. Friedrich, and M. Farge, 2012: Conditional vorticity budget of coherent and incoherent flow contributions in fully developed homogeneous isotropic turbulence. Phys. Fluids, 24, 035108, https://doi.org/10.1063/1.3694807.

Williams, A. G., and J. M. Hacker, 1992: The composite shape and structure of coherent eddies in the convective boundary layer. Bound.-Layer Meteor., 61, 213-245, https://doi.org/ 10.1007/BF02042933.

Wyngaard, J. C., 1982: Lectures on the planetary boundary layer. Mesoscale Meteorology-Theory, Observations and Models. D. K. Lilly and T. Gal-Chen, Eds., D. Reidel, 603-650.

2010: Turbulence in the Atmosphere. Cambridge University Press, $393 \mathrm{pp}$.

, and R. A. Brost, 1984: Top-down and bottom-up diffusion of a scalar in the convective boundary layer. J. Atmos. Sci., 41, 102-112, https://doi.org/10.1175/1520-0469(1984) 041<0102:TDABUD>2.0.CO;2.

, and J. C. Weil, 1991: Transport asymmetry in skewed turbulence. Phys. Fluids, 3A, 155-162, https://doi.org/10.1063/1.857874.

- and C.-H. Moeng, 1992: Parameterizing turbulent diffusion through the joint probability density. Bound.-Layer Meteor., 60, 1-13, https://doi.org/10.1007/BF00122059.

Xue, Y. K., and J. Shukla, 1993: The influence of land surface properties on Sahel climate. Part I: Desertification. J. Climate, 6, 2232-2245, https://doi.org/10.1175/1520-0442 (1993)006<2232:TIOLSP > 2.0.CO;2.

Yaglom, A. M., 1979: Similarity laws for constant-pressure and pressure-gradient turbulent wall flows. Annu. Rev. Fluid Mech., 11, 505-540, https://doi.org/10.1146/annurev.fl.11.010179.002445.

Young, G. S., 1988a: Turbulence structure of the convective boundary layer. Part I: Variability of normalized turbulence statistics. J. Atmos. Sci., 45, 719-726, https://doi.org/10.1175/ 1520-0469(1988)045<0719:TSOTCB >2.0.CO;2.

1988b: Turbulence structure of the convective boundary layer. Part II: Phoenix 78 aircraft observations of thermals and their environment. J. Atmos. Sci., 45, 727-735, https://doi.org/ 10.1175/1520-0469(1988)045<0727:TSOTCB > 2.0.CO;2.

Zhuang, Y., 1995: Dynamics and energetics of convective plumes in the atmospheric surface layer. J. Atmos. Sci., 52, 1712-1722, https://doi.org/10.1175/1520-0469(1995) 052<1712:DAEOCP $>2.0$. CO 2 .

Zilitinkevich, S. S., and Coauthors, 2006: The influence of large convective eddies on the surface-layer turbulence. Quart. J. Roy. Meteor. Soc., 132, 1423-1456, https://doi.org/10.1256/qj.05.79. 